\title{
Bloch Approximation in Homogenization and Applications*
}

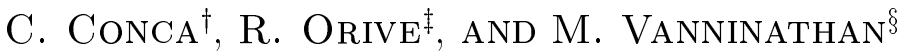

March 20, 2001

\begin{abstract}
The classical problem of homogenization of elliptic operators with periodically oscillating coefficients is revisited in this paper. As is well-known, homogenization process in classical framework is concerned with the study of asymptotic behaviour of solutions $u^{\varepsilon}$ of boundary value problems associated with such operators when the period $\varepsilon>0$ of the coefficients is small. In a previous work by C. CONCA AND M. VANNINATHAN [5], a new proof of weak convergence as $\varepsilon \rightarrow 0$ towards the homogenized solution was furnished using Bloch wave decomposition.

Following the same approach here, we go further and introduce what we call Bloch Approximation which will provide energy norm approximation for the solution $u^{\varepsilon}$. We develop several of its main features. As a simple application of this new object, we show that it contains both the first and second order correctors. Necessarily, the Bloch approximation will have to capture the oscillations of the solution in a sharper way. The present approach sheds new light and offers an alternative to view classical results.
\end{abstract}

Key words: homogenization, Bloch waves, correctors.

\section{Contents}

1 Introduction $\quad 2$

1.1 Survey of the previous results $\ldots \ldots \ldots \ldots \ldots \ldots$

1.2 Presentation of new results: The Bloch approximation . . . . . . . . . . 8

2 Fundamental lemmas $\quad 11$

3 Higher Bloch modes are negligible $\quad 16$

\footnotetext{
${ }^{*}$ This work has been partially supported by FondAP through its Programme on Mathematical-Mechanics.

${ }^{\dagger}$ Departamento de Ingeniería Matemática, Facultad de Ciencias Físicas y Matemáticas, Universidad de Chile, and Centro de Modelamiento Matemático, UMR 2071 CNRS-UChile, Casilla 170/3 - Correo 3, Santiago (CHILE).

${ }^{\ddagger}$ Departamento de Matemática Aplicada, Facultad de Ciencias Matemáticas, Universidad Complutense de Madrid, 28040 Madrid (EsPaña).

${ }^{\S}$ IISc-TIFR Mathematics Programme, TIFR Center, P.O. Box 1234, Bangalore - 560012 (INDIA).
} 
4.1 First order derivatives . . . . . . . . . . . . . . . . . . . . . . . . 19

4.2 Second order derivatives . . . . . . . . . . . . . . . . . . . . . . . . . . . 20

4.3 Third order derivatives . . . . . . . . . . . . . . . . . . . . . 20

4.4 Fourth order derivatives . . . . . . . . . . . . . . . . . . . . . . 21

5 Convergence of the first Bloch transform to Fourier transform $\quad 21$

6 Proof of the main convergence results 25

6.1 No concentration of energy at infinity . . . . . . . . . . . . . . . . . 25

6.2 Corrector result in $\mathbb{R}^{N}$. . . . . . . . . . . . . . . . . . . . . . . 27

6.3 Asymptotic expansion of the Bloch approximation . . . . . . . . . . . . 29

\section{Introduction}

In this paper, the classical problem of homogenization of elliptic operators with periodically oscillating coefficients is revisited. As is well known, homogenization process is concerned with the study of the behavior of solutions $u^{\varepsilon}$ of boundary value problems associated with such operators when the coefficients are periodic with small period $\varepsilon>0$. For an excellent introduction to this subject, the reader is referred to the book of A. Bensoussan, J.L. Lions and G. Papanicolaou [1]. In a previous work by C. CONCA AND M. VANNINATHAN [5], a new proof of weak convergence of $u^{\varepsilon}$ towards the homogenized solution $u^{*}$ was furnished using Bloch wave decomposition. Following the same approach, we go further and introduce what we call Bloch Approximation of the solution $u^{\varepsilon}$. As a simple application of this new object, we treat the problem of correctors in homogenization. At this point, it is worthwhile to remark that the homogenized solution $u^{*}$ is merely the weak limit of solutions $u^{\varepsilon}$ as $\varepsilon \rightarrow 0$. The idea behind introducing correctors is to look for terms (called first order correctors) which when added to the homogenized solution provide an approximation in the energy norm for all $\varepsilon$ sufficiently small. Second order correctors yield an error estimate in the energy norm of order $O(\varepsilon)$. The main feature of Bloch approximation is that it contains both the first and second order corrector terms. Another important feature is that it is easily computable in principle.

Historically, a classical way of obtaining such correctors is to work in the physical space and use multiscale expansion of the solution which was first introduced in the basic book just cited. As we will see, the method of Bloch waves sheds new light and offers an alternative to view the classical results. This method naturally leads us to work in the Fourier space, and thus in a framework dual to the one used in L. TARTAR [8]. However, it is important to mention that the Bloch wave method does not presuppose any multiscale structure of the solution; on the contrary, such a structure of the solution will be a consequence of the present method. Although correctors are generally not unique, our approach yields aposteriori the same ones as those obtained in [1].

Before proceeding further, we mention a word about the notations adopted in the sequel. Unless mentioned explicitly, the usual summation convention with respect to the repeated indices is understood. The constants appearing in various estimates independent of $\varepsilon$ are generically denoted by $c, c_{1}, c_{2}$ etc. Apart form the usual norms in Sobolev spaces $H^{1}, H^{2}$, we will also use the following semi-norms:

$$
|v|_{H^{1}}=\left\{\sum_{j=1}^{N}\left\|D_{j} v\right\|_{L^{2}}^{2}\right\}^{\frac{1}{2}}, \quad|v|_{H^{2}}=\left\{\sum_{j, k=1}^{N}\left\|D_{j, k}^{2} v\right\|_{L^{2}}^{2}\right\}^{\frac{1}{2}} .
$$


Let us now introduce the problem to be studied in this work. We consider the operator

$$
A \stackrel{\text { def }}{=}-\frac{\partial}{\partial y_{k}}\left(a_{k \ell}(y) \frac{\partial}{\partial y_{\ell}}\right), \quad y \in \mathbb{R}^{N}
$$

where the coefficients satisfy

$$
\left\{\begin{array}{l}
\left.a_{k \ell} \in L_{\#}^{\infty}(Y) \quad \text { where } \quad Y=\right] 0,2 \pi\left[^{N} \text {, i.e., each } a_{k \ell}\right. \text { is a } \\
Y \text {-periodic bounded measurable function defined on } \mathbb{R}^{N}, \text { and } \\
\exists \alpha>0 \quad \text { such that } a_{k \ell}(y) \eta_{k} \eta_{\ell} \geq \alpha|\eta|^{2} \quad \forall \eta \in \mathbb{R}^{N}, y \in Y \text { a.e., } \\
a_{k \ell}=a_{\ell k} \quad \forall k, \ell=1, \ldots, N .
\end{array}\right.
$$

For each $\varepsilon>0$, we consider also the operator $A^{\varepsilon}$ where

$$
A^{\varepsilon} \stackrel{\text { def }}{=}-\frac{\partial}{\partial x_{k}}\left(a_{k \ell}^{\varepsilon}(x) \frac{\partial}{\partial x_{\ell}}\right) \quad \text { with } \quad a_{k \ell}^{\varepsilon}(x)=a_{k \ell}\left(\frac{x}{\varepsilon}\right) \quad x \in \mathbb{R}^{N} .
$$

In homogenization theory, it is usual to refer to $x$ and $y$ the slow and the fast variables respectively. They are related by $y=\frac{x}{\varepsilon}$. Associated with $A^{\varepsilon}$, let us consider the following boundary-value problem

$$
A^{\varepsilon} u^{\varepsilon}=f \quad \text { in } \quad \Omega, \quad u^{\varepsilon} \in H_{0}^{1}(\Omega),
$$

which is posed in an arbitrary bounded domain $\Omega$ in $\mathbb{R}^{N}$ and $f$ is a given element in $L^{2}(\Omega)$. It is classical that the above problem admits one and only one solution.

From the classical work [1], it is known that one can associate to $A^{\varepsilon}$ a homogenized operator $A^{*}$ given by

$$
A^{*} \stackrel{\text { def }}{=}-\frac{\partial}{\partial x_{k}}\left(q_{k \ell} \frac{\partial}{\partial x_{\ell}}\right) \text {. }
$$

The homogenized coefficients $q_{k \ell}$ are constants and their definition is given below. The solution $u^{\varepsilon}$ of (1.4) converges weakly in $H_{0}^{1}(\Omega)$ to the so-called homogenized solution $u^{*}$ characterized by

$$
A^{*} u^{*}=f \quad \text { in } \quad \Omega, \quad u^{*} \in H_{0}^{1}(\Omega) .
$$

In the present paper, we do not consider the effects of boundaries postponing them to a subsequent article [3]. In the case of $\mathbb{R}^{N}$, it is natural to replace the operator $A^{\varepsilon}$ by $\left(A^{\varepsilon}+I\right)$. In that case, if $w^{\varepsilon}$ satisfies

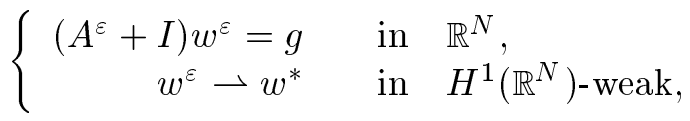

where $g$ is a given function in $L^{2}\left(\mathbb{R}^{N}\right)$, then it can be seen that (see Proposition 6.1 below)

$$
w^{\varepsilon} \rightarrow w^{*} \quad \text { in } \quad L^{2}\left(\mathbb{R}^{N}\right) \text {-strong. }
$$

In view of the above result, there is no concentration of $L^{2}$-energy at infinity and therefore, we will consider throughout this paper a sequence $u^{\varepsilon}$ and a function $f \in L^{2}\left(\mathbb{R}^{N}\right)$ satisfying

$$
\left\{\begin{array}{cll}
A^{\varepsilon} u^{\varepsilon}=f & \text { in } & \mathbb{R}^{N}, \\
u^{\varepsilon} \rightarrow u^{*} & \text { in } & H^{1}\left(\mathbb{R}^{N}\right) \text {-weak } \\
u^{\varepsilon} \rightarrow u^{*} & \text { in } & L^{2}\left(\mathbb{R}^{N}\right) \text {-strong. }
\end{array}\right.
$$


The central issue in the analysis of the first order correctors is to obtain functions $u_{1}^{\varepsilon} \in H^{1}\left(\mathbb{R}^{N}\right)$, which can be easily constructed and have the following characteristic property

$$
\left\|u^{\varepsilon}-u^{*}-\varepsilon u_{1}^{\varepsilon}\right\|_{H^{1}\left(\mathbb{R}^{N}\right)} \rightarrow 0 \quad \text { as } \quad \varepsilon \rightarrow 0
$$

By definition, second order correctors $u_{2}^{\varepsilon} \in H^{1}\left(\mathbb{R}^{N}\right)$ will enjoy the property

$$
\left\|u^{\varepsilon}-u^{*}-\varepsilon u_{1}^{\varepsilon}-\varepsilon^{2} u_{2}^{\varepsilon}\right\|_{H^{1}\left(\mathbb{R}^{N}\right)} \leq c \varepsilon .
$$

One of the purposes in this article is to carry out a more general construction than the classical one for correctors, namely Bloch approximation $\theta^{\varepsilon}$, which contains all the above correctors and justify the procedure. Apart from this, $\theta^{\varepsilon}$ contains a lot of information about the periodic medium which will be amply demonstrated in this paper.

\subsection{Survey of the previous results}

In the classical book [1] the authors obtain an asymptotic expansion (with $y=\frac{x}{\varepsilon}$ ) of the form

$$
\begin{aligned}
u^{\varepsilon}(x)=u^{*}(x) & +\varepsilon\left\{\chi_{k}(y) \frac{\partial u^{*}}{\partial x_{k}}(x)+\widetilde{u}_{1}(x)\right\}+ \\
+ & \varepsilon^{2}\left\{\chi_{k \ell}(y) \frac{\partial^{2} u^{*}}{\partial x_{k} x_{\ell}}(x)+\chi_{\ell}(y) \frac{\partial \widetilde{u}_{1}}{\partial x_{\ell}}(x)+\widetilde{u}_{2}(x)\right\}+\cdots
\end{aligned}
$$

Here, $\chi_{k}$ is the unique solution of the cell problem

$$
\left\{\begin{array}{l}
A \chi_{k}=\frac{\partial a_{k \ell}}{\partial y_{\ell}} \quad \text { in } \quad \mathbb{R}^{N} \\
\chi_{k} \in H_{\#}^{1}(Y), \quad \mathcal{M}_{Y}\left(\chi_{k}\right) \stackrel{\text { def }}{=} \frac{1}{|Y|} \int_{Y} \chi_{k} d y=0 .
\end{array}\right.
$$

The function $\chi_{k \ell}$ is characterized as the unique solution of

$$
\left\{\begin{array}{l}
A \chi_{k \ell}=a_{k \ell}+a_{k m} \frac{\partial \chi_{\ell}}{\partial y_{m}}-\frac{\partial}{\partial y_{m}}\left(a_{m k} \chi_{\ell}\right)-\mathcal{M}_{Y}\left(a_{k \ell}\right)-\mathcal{M}_{Y}\left(a_{k m} \frac{\partial \chi_{\ell}}{\partial y_{m}}\right) \text { in } \mathbb{R}^{N}, \\
\chi_{k \ell} \in H_{\#}^{1}(Y), \quad \mathcal{M}_{Y}\left(\chi_{k \ell}\right)=0 .
\end{array}\right.
$$

Further $\widetilde{u}_{1}(x), \widetilde{u}_{2}(x), \ldots$ are independent of $\varepsilon$ and satisfy equations of the type $A^{*} \widetilde{u}_{j}=\widetilde{g}_{j}$ in $\mathbb{R}^{N}$, where, for instance, $\widetilde{g}_{1}(x)=b_{j k \ell} D_{j k \ell}^{3} u^{*}$, where $b_{j k \ell}$ are constants:

$$
b_{j k \ell}=\mathcal{M}_{Y}\left(a_{j m} \frac{\partial \chi_{k \ell}}{\partial y_{m}}+a_{k \ell} \chi_{j}\right) \quad \forall j, k, \ell=1, \ldots, N .
$$

With these notations, the classical formula of the homogenized coefficients is as follows:

$$
q_{k \ell}=\mathcal{M}_{Y}\left(a_{k \ell}+a_{k m} \frac{\partial \chi_{\ell}}{\partial y_{m}}\right) \quad \forall k, \ell=1, \ldots, N .
$$

(another characterization of $q_{k \ell}$ is given in Proposition 1.5 below).

Using the above expansion, the first order corrector term is obtained in [1]. More precisely, we have 
Theorem 1.1 We assume that the coefficients a $a_{k \ell}$ satisfy assumptions (1.2), $f \in L^{2}\left(\mathbb{R}^{N}\right)$, and the solution $\chi_{k} \in W^{1, \infty}(Y), k=1, \ldots, N$. Then the first order corrector is defined by

$$
u_{1}^{\varepsilon}(x)=\chi_{k}\left(\frac{x}{\varepsilon}\right) \frac{\partial u^{*}}{\partial x_{k}}(x)
$$

which means that

$$
\left\|u^{\varepsilon}-u^{*}-\varepsilon u_{1}^{\varepsilon}\right\|_{H^{1}\left(\mathbb{R}^{N}\right)} \rightarrow 0 \quad \text { as } \quad \varepsilon \rightarrow 0 .
$$

In this paper, we obtain a more general result using a different approach introduced in [5]. The basic tool of this new approach is Bloch waves $\psi$ associated with $A$ which we define now. Let us consider the following spectral problem parameterized by $\eta \in \mathbb{R}^{N}$ : find $\lambda=\lambda(\eta) \in \mathbb{R}$ and $\psi=\psi(y ; \eta)$ (not identically zero) such that

$$
\left\{\begin{array}{l}
A \psi(\cdot ; \eta)=\lambda(\eta) \psi(\cdot ; \eta) \text { in } \mathbb{R}^{N}, \quad \psi(\cdot ; \eta) \text { is }(\eta ; Y) \text {-periodic, i.e. } \\
\psi(y+2 \pi m ; \eta)=e^{2 \pi i m \cdot \eta} \psi(y ; \eta) \quad \forall m \in \mathbb{Z}^{N}, y \in \mathbb{R}^{N}
\end{array}\right.
$$

Next, we define $\phi(y ; \eta)=e^{-i y \cdot \eta} \psi(y ; \eta)$ and (1.15) can be rewritten in terms of $\phi$ as follows:

$$
A(\eta) \phi=\lambda \phi \quad \text { in } \quad \mathbb{R}^{N}, \quad \phi \text { is } Y \text {-periodic. }
$$

Here, the operator $A(\eta)$ is defined by

$$
A(\eta) \stackrel{\text { def }}{=}-\left(\frac{\partial}{\partial y_{k}}+i \eta_{k}\right)\left[a_{k \ell}(y)\left(\frac{\partial}{\partial y_{\ell}}+i \eta_{\ell}\right)\right]
$$

which can be rewritten as

$$
A(\eta)=A+i \eta_{k} C_{k}+\eta_{k} \eta_{\ell} a_{k \ell}(y)
$$

with

$$
C_{k} \phi \stackrel{\text { def }}{=}-a_{k j}(y) \frac{\partial \phi}{\partial y_{j}}-\frac{\partial}{\partial y_{j}}\left(a_{k j}(y) \phi\right) .
$$

It is clear from (1.15) that the $(\eta, Y)$ periodicity condition is unaltered if we replace $\eta$ by $(\eta+q)$ with $q \in \mathbb{Z}^{N}$ and $\eta$ can therefore be confined to the dual cell $\eta \in Y^{\prime}=\left[-\frac{1}{2}, \frac{1}{2}{ }^{N}\right.$. It is well known (Conca, Planchard and Vanninathan [4]) that for each $\eta \in Y^{\prime}$, the above spectral problem admits a discrete sequence of eigenvalues with the following properties:

$$
\left\{\begin{array}{l}
0 \leq \lambda_{1}(\eta) \leq \cdots \leq \lambda_{m}(\eta) \leq \cdots \rightarrow \infty \\
\forall m \geq 1, \lambda_{m}(\eta) \text { is a Lipschitz function of } \eta \in Y^{\prime} .
\end{array}\right.
$$

Besides, the corresponding eigenfunctions denoted by $\psi_{m}(\cdot ; \eta)$ and $\phi_{m}(\cdot ; \eta)$ form orthonormal bases in the spaces of all $L_{l o c}^{2}\left(\mathbb{R}^{N}\right)$-functions which are $(\eta ; Y)$-periodic and $Y$-periodic respectively; these spaces are denoted by $L_{\#}^{2}(\eta ; Y)$ and $L_{\#}^{2}(Y)$. It is worthwhile to remark that these eigenfunctions belong in fact to the spaces $H_{\#}^{1}(\eta ; Y)$ and $H_{\#}^{1}(Y)$ respectively, where

$$
\begin{aligned}
H_{\#}^{1}(\eta ; Y) & =\left\{\psi \in L_{\#}^{2}(\eta ; Y) \mid \frac{\partial \psi}{\partial y_{k}} \in L_{\#}^{2}(\eta ; Y) \quad \forall k=1, \ldots, N\right\}, \\
H_{\#}^{1}(Y) & =\left\{\phi \in L_{\#}^{2}(Y) \mid \frac{\partial \phi}{\partial y_{k}} \in L_{\#}^{2}(Y) \quad \forall k=1, \ldots, N\right\} .
\end{aligned}
$$

The functions $\psi_{m}(\cdot ; \eta)$ and $\phi_{m}(\cdot ; \eta)$ (referred to as Bloch waves) introduced above enable us to describe the spectral resolution of $A$ (an unbounded self-adjoint operator in $L^{2}\left(\mathbb{R}^{N}\right)$ ) in the orthogonal basis $\left\{e^{i y \cdot \eta} \phi_{m}(y ; \eta) \mid m \geq 1, \eta \in Y^{\prime}\right\}$. More precisely, we have 
Theorem 1.2 Let $g \in L^{2}\left(\mathbb{R}^{N}\right)$. The $m^{\text {th }}$ Bloch coefficient of $g$ is defined as follows:

$$
\left(B_{m} g\right)(\eta)=\int_{\mathbb{R}^{N}} g(y) e^{-i y \cdot \eta} \bar{\phi}_{m}(y ; \eta) d y \quad \forall m \geq 1, \eta \in Y^{\prime} .
$$

Then the following inverse formula holds:

$$
g(y)=\int_{Y^{\prime}} \sum_{m=1}^{\infty}\left(B_{m} g\right)(\eta) e^{i y \cdot \eta} \phi_{m}(y ; \eta) d \eta .
$$

Further, we have Parseval's identity:

$$
\int_{\mathbb{R}^{N}}|g(y)|^{2} d y=\int_{Y^{\prime}} \sum_{m=1}^{\infty}\left|\left(B_{m} g\right)(\eta)\right|^{2} d \eta .
$$

Finally, for all $g$ in the domain of $A$, we have

$$
A g(y)=\int_{Y^{\prime}} \sum_{m=1}^{\infty} \lambda_{m}(\eta)\left(B_{m} g\right)(\eta) e^{i y \cdot \eta} \phi_{m}(y ; \eta) d \eta .
$$

To obtain the spectral resolution of $A^{\varepsilon}$ in an analogous manner, let us introduce Bloch waves at the $\varepsilon$-scale:

$$
\lambda_{m}^{\varepsilon}(\xi)=\varepsilon^{-2} \lambda_{m}(\eta), \quad \phi_{m}^{\varepsilon}(x ; \xi)=\phi_{m}(y ; \eta), \quad \psi_{m}^{\varepsilon}(x ; \xi)=\psi_{m}(y ; \eta),
$$

where the variables $(x, \xi)$ and $(y, \eta)$ are related by $y=\frac{x}{\varepsilon}$ and $\eta=\varepsilon \xi$. Observe that $\phi_{m}^{\varepsilon}(x ; \xi)$ is $\varepsilon Y$-periodic (in $x$ ) and $\varepsilon^{-1} Y^{\prime}$ periodic with respect to $\xi$. In the same manner, $\psi_{m}^{\varepsilon}(\cdot ; \xi)$ is $(\varepsilon \xi ; \varepsilon Y)$ periodic because of the relation $\psi_{m}^{\varepsilon}(x ; \xi)=e^{i x \cdot \xi} \phi_{m}^{\varepsilon}(x ; \xi)$. Note that the dual cell at $\varepsilon$-scale is $\varepsilon^{-1} Y^{\prime}$ and hence we take $\xi$ to vary in $\varepsilon^{-1} Y^{\prime}$ in the sequel. With these notations, we have the following result analogous to Theorem 1.2.

Theorem 1.3 Let $g \in L^{2}\left(\mathbb{R}^{N}\right)$. The $m^{\text {th }}$ Bloch coefficient of $g$ at the $\varepsilon$-scale is defined as follows:

$$
\left(B_{m}^{\varepsilon} g\right)(\xi)=\int_{\mathbb{R}^{N}} g(x) e^{-i x \cdot \xi} \bar{\phi}_{m}^{\varepsilon}(x ; \xi) d x \quad \forall m \geq 1, \xi \in \varepsilon^{-1} Y^{\prime} .
$$

Then the following inverse formula and Parseval's identity hold:

$$
\begin{array}{r}
g(x)=\int_{\varepsilon^{-1} Y^{\prime}} \sum_{m=1}^{\infty}\left(B_{m}^{\varepsilon} g\right)(\xi) e^{i x \cdot \xi} \phi_{m}^{\varepsilon}(x ; \xi) d \xi, \\
\int_{\mathbb{R}^{N}}|g(x)|^{2} d x=\int_{\varepsilon^{-1} Y^{\prime}} \sum_{m=1}^{\infty}\left|\left(B_{m}^{\varepsilon} g\right)(\xi)\right|^{2} d \xi .
\end{array}
$$

Finally, for all $g$ in the domain of $A^{\varepsilon}$, we get

$$
A^{\varepsilon} g(x)=\int_{\varepsilon^{-1} Y^{\prime}} \sum_{m=1}^{\infty} \lambda_{m}^{\varepsilon}(\xi)\left(B_{m}^{\varepsilon} g\right)(\xi) e^{i x \cdot \xi} \phi_{m}^{\varepsilon}(x ; \xi) d \xi .
$$

Using the above theorem, the classical homogenization result was deduced in [5]. Let us recall the main steps. The first one consists of considering a sequence $u^{\varepsilon} \in H^{1}\left(\mathbb{R}^{N}\right)$ satisfying (1.9). We can express the equation $A^{\varepsilon} u^{\varepsilon}=f$ in $\mathbb{R}^{N}$ in the equivalent form

$$
\lambda_{m}^{\varepsilon}(\xi)\left(B_{m}^{\varepsilon} u^{\varepsilon}\right)(\xi)=\left(B_{m}^{\varepsilon} f\right)(\xi) \quad \forall m \geq 1, \xi \in \varepsilon^{-1} Y^{\prime} .
$$

In the homogenization process, one can neglect all the relations for $m \geq 2$. More precisely, it is proved in [5] that the following result holds. 
Proposition 1.4 Let

$$
v^{\varepsilon}(x)=\int_{\varepsilon^{-1} Y^{\prime}} \sum_{m=2}^{\infty}\left(B_{m}^{\varepsilon} u^{\varepsilon}\right)(\xi) e^{i x \cdot \xi} \phi_{m}^{\varepsilon}(x ; \xi) d \xi .
$$

Then $\left\|v^{\varepsilon}\right\|_{L^{2}\left(\mathbb{R}^{N}\right)} \leq c \varepsilon$.

Thus we can concentrate our attention only on the relation corresponding to the first Bloch wave:

$$
\lambda_{1}^{\varepsilon}(\xi)\left(B_{1}^{\varepsilon} u^{\varepsilon}\right)(\xi)=\left(B_{1}^{\varepsilon} f\right)(\xi) \quad \forall \xi \in \varepsilon^{-1} Y^{\prime} .
$$

The homogenized equation in the Fourier space

$$
q_{k \ell} \xi_{k} \xi_{\ell} \widehat{u *}(\xi)=\widehat{f}(\xi) \quad \forall \xi \in \mathbb{R}^{N}
$$

is obtained from (1.22) by passing to the limit as $\varepsilon \rightarrow 0$. Here, the symbol ${ }^{\wedge}$ stands for the classical Fourier transformation

$$
\widehat{f}(\xi)=\frac{1}{(2 \pi)^{N / 2}} \int_{\mathbb{R}^{N}} f(x) e^{-i x \cdot \xi} d x .
$$

To this end, the following results were established and applied in [5]:

Proposition 1.5 We assume that $a_{k \ell}$ satisfy (1.2). Then there exists $\delta>0$ such that the first eigenvalue $\lambda_{1}(\eta)$ is an analytic function on $B_{\delta} \stackrel{\text { def }}{=}\{\eta|| \eta \mid<\delta\}$, and there is a choice of the first eigenvector $\phi_{1}(y ; \eta)$ satisfying

$$
\left\{\begin{array}{l}
\eta \rightarrow \phi_{1}(\cdot ; \eta) \in H_{\#}^{1}(Y) \text { is analytic on } B_{\delta}, \\
\phi_{1}(y ; 0)=p^{(0)}\left(=|Y|^{-1 / 2}=\frac{1}{(2 \pi)^{N / 2}}\right) .
\end{array}\right.
$$

Moreover, we have the relations

$$
\begin{gathered}
\lambda_{1}(0)=0, \quad D_{k} \lambda_{1}(0)=\frac{\partial \lambda_{1}}{\partial \eta_{k}}(0)=0 \quad \forall k=1, \ldots, N, \\
\frac{1}{2} D_{k \ell}^{2} \lambda_{1}(0)=\frac{1}{2} \frac{\partial^{2} \lambda_{1}}{\partial \eta_{k} \partial \eta_{\ell}}(0)=q_{k \ell} \quad \forall k, \ell=1, \ldots, N,
\end{gathered}
$$

and there exist constants $c$ and $\widetilde{c}$ such that

$$
\begin{aligned}
c|\eta|^{2} \leq \lambda_{1}(\eta) & \leq \widetilde{c}|\eta|^{2} \quad \forall \eta \in Y^{\prime}, \\
0 & <\lambda_{2}^{(N)} \leq \lambda_{m}(\eta) \quad \forall m \geq 2, \eta \in Y^{\prime},
\end{aligned}
$$

where $\lambda_{2}^{(N)}$ is the second eigenvalue of the spectral problem for $A$ in the cell $Y$ with Neumann boundary conditions on $\partial Y$.

Apart from the above result of regularity on the Bloch spectrum, we need to prove that the first Bloch transform is an approximation to Fourier transform. This result is naturally expected from the fact that $\phi_{1}^{\varepsilon}(x ; \xi) \rightarrow(2 \pi)^{-N / 2}$, as $\varepsilon \rightarrow 0, \forall \xi \in \mathbb{R}^{N}$.

Proposition 1.6 Let $g^{\varepsilon}$ and $g$ be in $L^{2}\left(\mathbb{R}^{N}\right)$. Then

(i) If $g^{\varepsilon} \rightarrow g$ weakly in $L^{2}\left(\mathbb{R}_{x}^{N}\right)$, then $\chi_{\varepsilon^{-1} Y^{\prime}} B_{1}^{\varepsilon} g^{\varepsilon} \rightarrow \widehat{g}$ weakly in $L_{l o c}^{2}\left(\mathbb{R}_{\xi}^{N}\right)$ provided there is a fixed compact set $K$ such that $\operatorname{supp}\left(g^{\varepsilon}\right) \subset K, \forall \varepsilon$.

(ii) If $g^{\varepsilon} \rightarrow g$ in $L^{2}\left(\mathbb{R}_{x}^{N}\right)$, then $\chi_{\varepsilon^{-1} Y^{\prime}} B_{1}^{\varepsilon} g^{\varepsilon} \rightarrow \widehat{g}$ in $L_{l o c}^{2}\left(\mathbb{R}_{\xi}^{N}\right)$.

These results easily lead us to the following homogenization theorem in $\mathbb{R}^{N}$ : 
Theorem 1.7 We consider a sequence $u^{\varepsilon}$ satisfying (1.9). Then

$$
a_{k \ell}^{\varepsilon} \frac{\partial u^{\varepsilon}}{\partial x_{\ell}} \rightarrow q_{k \ell} \frac{\partial u^{*}}{\partial x_{\ell}} \quad \text { in } \quad L^{2}\left(\mathbb{R}^{N}\right), \quad \forall k=1, \ldots, N .
$$

In particular, $u^{*}$ satisfies $A^{*} u^{*}=f$ in $\mathbb{R}^{N}$.

Once the homogenization result in $\mathbb{R}^{N}$ is established, it is an easy matter to deduce the corresponding result in a bounded domain $\Omega$ by localization techniques using a cut-off function $\phi \in \mathcal{D}(\Omega)$ (see $[5])$.

\subsection{Presentation of new results: The Bloch approximation}

Let us consider the sequence $u^{\varepsilon}$ satisfying hypotheses (1.9). The Bloch Approximation of $u^{\varepsilon}$ is defined by the following formula:

$$
\theta^{\varepsilon}(x) \stackrel{\text { def }}{=} \int_{\varepsilon^{-1} Y^{\prime}} \widehat{u^{*}}(\xi) e^{i x \cdot \xi} \phi_{1}^{\varepsilon}(x ; \xi) d \xi, \quad x \in \mathbb{R}^{N} .
$$

First of all, let us remark that this object is not difficult to be computed in principle. Our goal throughout this paper is to study properties of this function and particularly its relations with various correctors terms. It is worth noticing that $\theta^{\varepsilon}$ is defined only in terms of the first Bloch mode $\phi_{1}^{\varepsilon}$. We will see in Section 3 that higher Bloch modes $\phi_{m}^{\varepsilon}, m \geq 2$ do not contribute at all in the analysis of the correctors of first and second order in the energy norm. (It will be interesting to know whether these higher order modes play a part in the analysis of correctors in stronger norms $H^{2}, \ldots$ etc. For $H^{2}$ estimates, we refer to our work [6]). Thus we are motivated to introduce the projection onto the first Bloch mode: for all $g \in L^{2}\left(\mathbb{R}^{N}\right)$, we define

$$
P_{1}^{\varepsilon} g(x)=\int_{\varepsilon^{-1} Y^{\prime}} B_{1}^{\varepsilon} g(\xi) e^{i x \cdot \xi} \phi_{1}^{\varepsilon}(x ; \xi) d \xi, \quad x \in \mathbb{R}^{N} .
$$

We note by the item $(i i)$ of the Proposition 1.6 that the Fourier transform $\widehat{u *}$ is an approximation of $B_{1}^{\varepsilon} u^{\varepsilon}$. Therefore, heuristically speaking, the Bloch approximation $\theta^{\varepsilon}$ is close to $P_{1}^{\varepsilon} u^{\varepsilon}$, and hence to $u^{\varepsilon}$. With these notations, we will prove

Theorem 1.8 Assume that the coefficients $a_{k \ell}$ satisfy (1.2). Let $u^{\varepsilon}$ be the sequence introduced in (1.9). Then if $f \in L^{2}\left(\mathbb{R}^{N}\right)$, we have

$$
\left(u^{\varepsilon}-\theta^{\varepsilon}\right) \rightarrow 0 \quad \text { in } \quad H^{1}\left(\mathbb{R}^{N}\right) .
$$

Furthermore, we have the estimate

$$
\left|u^{\varepsilon}-\theta^{\varepsilon}\right|_{H^{1}\left(\mathbb{R}^{N}\right)} \leq c \varepsilon\|f\|_{L^{2}\left(\mathbb{R}^{N}\right)} .
$$

It is worth remarking that even though error estimates of the type (1.29) are sometimes found in the literature, they are usually obtained using maximum principle with more regularity hypotheses on $a_{k \ell}$ and $f$. Here, we obtain these natural estimates under optimal hypotheses.

Thanks to the above result, we are reduced to expand $\theta^{\varepsilon}$ in terms of $\varepsilon$ in order to be able to compare it with the classical correctors for $u^{\varepsilon}$. To fulfill this task, it is clear from the definition of $\theta^{\varepsilon}$, that it is necessary to obtain asymptotic expansions of the first eigenvalue $\lambda_{1}^{\varepsilon}(\xi)$, and the first 
Bloch mode $\phi_{1}^{\varepsilon}(\cdot ; \xi)$. (In addition, for our purposes below, we need an asymptotic expansion of the first Bloch transform $B_{1}^{\varepsilon} g(\xi)$ for which we refer the reader to Section 5. These results strengthen previous ones, in particular, Proposition 1.6). We state now results in this direction and their proofs will be taken up in the following sections along with other auxiliary results. First, we introduce some test functions $\chi_{k \ell}, \chi_{k \ell m}, \chi_{k \ell m n}$ defined by the following cell problems (observe that the first ones of them are nothing but the functions already introduced in (1.14)):

$$
\left\{\begin{array}{l}
A \chi_{k \ell}=\left(a_{k \ell}-q_{k \ell}\right)-\frac{1}{2}\left(C_{k} \chi_{\ell}+C_{\ell} \chi_{k}\right) \quad \text { in } \mathbb{R}^{N}, \\
\chi_{k \ell} \in H_{\#}^{1}(Y), \quad \mathcal{M}_{Y}\left(\chi_{k \ell}\right)=0 .
\end{array}\right.
$$

$$
\begin{aligned}
& \left\{\begin{array}{c}
A \chi_{k \ell m}=\frac{1}{3}\left[\left(a_{k \ell}-q_{k \ell}\right) \chi_{m}+\left(a_{\ell m}-q_{\ell m}\right) \chi_{k}+\left(a_{m k}-q_{m k}\right) \chi_{\ell}-C_{k} \chi_{\ell m}-C_{\ell} \chi_{m k}-\right. \\
\left.-C_{m} \chi_{k \ell}\right] \text { in } \mathbb{R}^{N}, \\
\chi_{k \ell m} \in H_{\#}^{1}(Y), \quad \mathcal{M}_{Y}\left(\chi_{k \ell m}\right)=0 .
\end{array}\right. \\
& \left\{\begin{array}{c}
A \chi_{k \ell m n}=\frac{1}{4 !} D_{k \ell m n}^{4} \lambda_{1}(0)-\frac{1}{4}\left(C_{n} \chi_{k \ell m}+C_{k} \chi_{\ell m n}+C_{\ell} \chi_{m n k}+C_{m} \chi_{n k \ell}\right)+ \\
+\frac{1}{3 !}\left[\left(a_{k \ell}-q_{k \ell}\right) \chi_{m n}+\left(a_{\ell m}-q_{\ell m}\right) \chi_{k n}+\left(a_{k m}-q_{k m}\right) \chi_{\ell n}+\right. \\
\left.+\left(a_{m n}-q_{m n}\right) \chi_{k \ell}+\left(a_{\ell n}-q_{\ell n}\right) \chi_{k m}+\left(a_{k n}-q_{k n}\right) \chi_{\ell m}\right] \text { in } \mathbb{R}^{N}, \\
\chi_{k \ell m n} \in H_{\#}^{1}(Y), \quad \mathcal{M}_{Y}\left(\chi_{k \ell m n}\right)=0 .
\end{array}\right.
\end{aligned}
$$

Proposition 1.9 All odd order derivatives of $\lambda_{1}$ at $\eta=0$ vanish, i.e.,

$$
D^{\beta} \lambda_{1}(0)=0 \quad \forall \beta \in \mathbb{Z}_{+}^{N},|\beta| \text { odd } .
$$

All even order derivatives of $\lambda_{1}$ at $\eta=0$ can be calculated in a systematic fashion. For instance, the fourth order derivatives have the following expressions: for all $k, \ell, m, n=1, \ldots, N$

$$
\begin{array}{r}
\frac{1}{4 !} D_{k \ell m n}^{4} \lambda_{1}(0)=\frac{1}{4} \frac{1}{|Y|} \int_{Y}\left\{C_{n} \chi_{k \ell m}+C_{k} \chi_{\ell m n}+C_{\ell} \chi_{m n k}+C_{m} \chi_{n k \ell}\right\} d y- \\
-\frac{1}{3 !} \frac{1}{|Y|} \int_{Y}\left\{\left(a_{k \ell}-q_{k \ell}\right) \chi_{m n}+\left(a_{\ell m}-q_{\ell m}\right) \chi_{n k}+\left(a_{m n}-q_{m n}\right) \chi_{k \ell}+\right. \\
\left.+\left(a_{n k}-q_{n k}\right) \chi_{\ell m}+\left(a_{k m}-q_{k m}\right) \chi_{\ell n}+\left(a_{\ell n}-q_{\ell n}\right) \chi_{k m}\right\} d y
\end{array}
$$

Various derivatives of $\phi_{1}$ at $\eta=0$ can also be calculated in a systematic fashion.

Proposition 1.10 We have the following expressions

$$
\begin{aligned}
D_{k} \phi_{1}(y ; 0)= & i p^{(0)} \chi_{k}(y), \\
\frac{1}{2 !} D_{k \ell}^{2} \phi_{1}(y ; 0)= & -p^{(0)} \chi_{k \ell}(y)-\beta_{k \ell}^{(2)} p^{(0)}, \\
\frac{1}{3 !} D_{k \ell m}^{3} \phi_{1}(y ; 0)= & -i p^{(0)} \chi_{k \ell m}(y)-\frac{i}{3}\left(\beta_{k \ell}^{(2)} \chi_{m}(y)+\beta_{\ell m}^{(2)} \chi_{k}(y)+\beta_{m k}^{(2)} \chi_{\ell}(y)\right) p^{(0)}, \\
\frac{1}{4 !} D_{k \ell m n}^{4} \phi_{1}(y ; 0)= & p^{(0)} \chi_{k \ell m n}(y)-\frac{1}{3 !}\left(\beta_{k \ell}^{(2)} \chi_{m n}(y)+\beta_{\ell m}^{(2)} \chi_{n k}(y)+\beta_{m n}^{(2)} \chi_{k \ell}+\right. \\
& \left.+\beta_{n k}^{(2)} \chi_{\ell m}(y)+\beta_{k m}^{(2)} \chi_{n \ell}(y)+\beta_{\ell n}^{(2)} \chi_{k m}(y)\right) p^{(0)}+\beta_{k \ell m n}^{(4)} p^{(0)}
\end{aligned}
$$


with

$$
\begin{aligned}
\beta_{k \ell}^{(2)}= & \frac{1}{2 !} \frac{1}{|Y|} \int_{Y} \chi_{\ell} \chi_{k} d y \\
\beta_{k \ell m n}^{(4)}= & \frac{1}{|Y|} \int_{Y} \frac{1}{4}\left[\chi_{\ell m n} \chi_{k}+\chi_{k m n} \chi_{\ell}+\chi_{n \ell k} \chi_{m}+\chi_{k \ell n} \chi_{n}\right] d y- \\
& -\frac{1}{|Y|} \int_{Y} \frac{1}{6}\left[\chi_{\ell m} \chi_{k n}+\chi_{k m} \chi_{n \ell}+\chi_{\ell k} \chi_{n m}\right] d y+ \\
& +\frac{1}{|Y|} \frac{1}{2}\left(\beta_{k \ell}^{(2)} \beta_{m n}^{(2)}+\beta_{k m}^{(2)} \beta_{n \ell}^{(2)}+\beta_{k n}^{(2)} \beta_{m \ell}^{(2)}\right)
\end{aligned}
$$

We note that all odd order derivatives of $\phi_{1}$ at $\eta=0$ are purely imaginary and all even order derivatives are real.

Since $\phi_{1}(\cdot ; \eta)$ is proved to be analytic for $|\eta| \leq \delta$, we can expand it and this gives rise to an asymptotic expansion of $\theta^{\varepsilon}$ which is as follows:

$$
\theta^{\varepsilon}(x)=u^{*}(x)+\varepsilon \chi_{k}\left(\frac{x}{\varepsilon}\right) \frac{\partial u^{*}}{\partial x_{k}}(x)-\varepsilon^{2}\left(\chi_{k \ell}\left(\frac{x}{\varepsilon}\right)+\beta_{k \ell}^{(2)}\right) \frac{\partial^{2} u^{*}}{\partial x_{k} \partial x_{\ell}}(x)+\cdots
$$

This can be rigorously proved. Our next result is a sample where we specify the precise hypotheses needed to justify the above expansion up to three terms.

Theorem 1.11 Assume that the hypotheses of Theorem 1.8 hold.

(i) If $u^{*} \in H^{1}\left(\mathbb{R}^{N}\right)$, then

$$
\left\|\theta^{\varepsilon}-u^{*}\right\|_{L^{2}\left(\mathbb{R}^{N}\right)} \leq c \varepsilon\left\|u^{*}\right\|_{H^{1}\left(\mathbb{R}^{N}\right)} .
$$

(ii) If $f \in L^{2}\left(\mathbb{R}^{N}\right)$ and $\chi_{k} \in W_{\#}^{1, \infty}(Y)$ where $\chi_{k}$ is the solution of (1.13) and $\chi_{k}^{\varepsilon}(x)=\chi_{k}\left(\frac{x}{\varepsilon}\right)$, then we have

$$
\left\|\theta^{\varepsilon}-u^{*}-\varepsilon \chi_{k}^{\varepsilon} \frac{\partial u^{*}}{\partial x_{k}}\right\|_{H^{1}\left(\mathbb{R}^{N}\right)} \leq c \varepsilon\|f\|_{L^{2}\left(\mathbb{R}^{N}\right)} .
$$

(iii) If $f \in H^{1}\left(\mathbb{R}^{N}\right)$ and $\chi_{k}, \chi_{k \ell} \in W_{\#}^{1, \infty}(Y)$ where $\chi_{k \ell}$ is the solution of (1.30), $\beta_{k \ell}^{(2)}$ are constants defined in Proposition 1.10 and $\chi_{k \ell}^{\varepsilon}(x)=\chi_{k \ell}\left(\frac{x}{\varepsilon}\right)$, then

$$
\left\|\theta^{\varepsilon}-u^{*}-\varepsilon \chi_{k}^{\varepsilon} \frac{\partial u^{*}}{\partial x_{k}}+\varepsilon^{2}\left(\chi_{k \ell}^{\varepsilon}+\beta_{k \ell}^{(2)}\right) \frac{\partial^{2} u^{*}}{\partial x_{k} \partial x_{\ell}}\right\|_{H^{1}\left(\mathbb{R}^{N}\right)} \leq c \varepsilon^{2}\|f\|_{H^{1}\left(\mathbb{R}^{N}\right)} .
$$

It is important to note that these above expansions are of Taylor type owing to the analyticity of $\lambda_{1}(\eta)$ and $\phi_{1}(\cdot ; \eta)$. This is the main difference between this approach and the classical one found in [1] where the expansion has a multiscale structure.

The expansions of $\lambda_{1}(\eta), \phi_{1}^{\varepsilon}(\cdot ; \eta)$, and $B_{1}^{\varepsilon} g(\xi)$ obtained in Propositions 1.5, 1.9, and Propositions 5.1, 5.2, and 5.3 below has further interesting consequences which will be developed in a forthcoming paper. For the time being, we will be content with a few remarks. Since higher order modes can be neglected, the first eigenvalue $\lambda_{1}(\eta)$ along with the first eigenvector $\phi_{1}(\cdot ; \eta)$ represent the periodic medium under consideration. Their contributions occur somewhat separetely without interaction at the levels of homogenized equation and correctors. More precisely, the first eigenvalue 
$\lambda_{1}(\eta)$ contributes at various levels through its derivatives at $\eta=0$. The first eigenvector $\phi_{1}(\cdot ; \eta)$ and its first derivatives contribute through the first Bloch transform $B_{1}^{\varepsilon} g(\xi)$ and its expansion described in Propositions 5.2, 5.3.

In the homogenized equation, for instance, we see the product of the second order derivatives of $\lambda_{1}(\eta)$ at $\eta=0$ with the $0^{\text {th }}$ order term of $B_{1}^{\varepsilon} g(\xi)$, namely $\widehat{g}(\xi)$. We see a similar structure in the correctors too. There seem to be situations where both interact and contribute jointly in a manner different from the above. One example of such a situation is the study of the propagation of waves in a periodic medium. It appears that the homogenized medium is not good enough to provide an approximation to the propagation for large times because of the appearance of dispersion effects shown numerically in F. SANTOSA AND W.W Symes [7]. We feel that this is an appropriate place to highlight the improvements achieved in this work with respect to [7]. Apart from the mathematical rigor, the main point is that the third order derivatives of $\lambda_{1}(\eta)$ at $\eta=0$ are shown to be zero even in the multi-dimensional case. (In fact all odd order derivatives vanish). Moreover, our arguments are more general compared with the one-dimensional case covered in [7]. This will have consequences in the propagation of waves in periodic media. We plan to cover these aspects in a future publication.

We conclude this Introduction by saying how the rest of this paper is organized. Section 2 is devoted to certain fundamental lemmas which are indispensable. As an immediate application, we prove, in Section 3, that the higher order Bloch modes are negligible. Taylor expansion for $\lambda_{1}$ and $\phi_{1}$ are obtained in Section 4 which proves Propositions 1.9 and 1.10. Section 5 is devoted to the description of the asymptotic behavior of the first Bloch transform $B_{1}^{\varepsilon}$ whose definition is given in Theorem 1.2. Finally, in Section 6, we present the proofs of the main results, namely Theorem 1.8 and Theorem 1.11.

\section{Fundamental lemmas}

In this section, we prove a series of results which generalize the Parseval's identity stated in Theorem 1.3. These estimates will be useful in the sequel for the analysis of correctors. The following two lemmas are easily seen to be generalizations of well-known classical results for $-\Delta$.

Lemma 2.1 For all $g \in H^{1}\left(\mathbb{R}^{\mathbb{N}}\right)$, we have

$$
c_{1}|g|_{H^{1}\left(\mathbb{R}^{N}\right)}^{2} \leq \int_{\varepsilon^{-1} Y^{\prime}} \sum_{m=1}^{\infty} \lambda_{m}^{\varepsilon}(\xi)\left|B_{m}^{\varepsilon} g(\xi)\right|^{2} d \xi \leq c_{2}|g|_{H^{1}\left(\mathbb{R}^{N}\right)}^{2}
$$

where $c_{1}$ and $c_{2}$ are constants independent of $\varepsilon$ and $g$.

Proof. First of all, by uniform ellipticity of $A^{\varepsilon}$, we have

$$
\alpha \int_{\mathbb{R}^{N}}|\nabla g|^{2} d x \leq \int_{\mathbb{R}^{N}} A^{\varepsilon} g \bar{g} d x \leq \beta \int_{\mathbb{R}^{N}}|\nabla g|^{2} d x .
$$

We can rewrite the middle term by applying Plancherel identity:

$$
\int_{\mathbb{R}^{N}} g(x) \overline{h(x)} d x=\int_{\varepsilon^{-1 Y^{\prime}}} \sum_{m=1}^{\infty} B_{m}^{\varepsilon} g(\xi) \overline{B_{m}^{\varepsilon} h(\xi)} d \xi \quad \forall g, h \in L^{2}\left(\mathbb{R}^{N}\right) .
$$


Indeed, using the spectral resolution of $A^{\varepsilon}$, we get

$$
\int_{\mathbb{R}^{N}} A^{\varepsilon} g \bar{g} d x=\int_{\varepsilon^{-1} Y^{\prime}} \sum_{m=1}^{\infty} \lambda_{m}^{\varepsilon}(\xi)\left|B_{m}^{\varepsilon} g(\xi)\right|^{2} d \xi .
$$

This completes the proof.

By using the duality between $H^{1}\left(\mathbb{R}^{N}\right)$ and $H^{-1}\left(\mathbb{R}^{N}\right)$, we deduce

Lemma 2.2 For all $g \in H^{-1}\left(\mathbb{R}^{N}\right)$, there exist $c_{1}$ and $c_{2}$ which are independent of $\varepsilon$ and $g$, such that

$$
c_{1}\|g\|_{H^{-1}\left(\mathbb{R}^{N}\right)}^{2} \leq \int_{\varepsilon^{-1} Y^{\prime}} \sum_{m=1}^{\infty} \frac{1}{1+\lambda_{m}^{\varepsilon}(\xi)}\left|B_{m}^{\varepsilon} g(\xi)\right|^{2} d \xi \leq c_{2}\|g\|_{H^{-1}\left(\mathbb{R}^{N}\right)}^{2} .
$$

In our next lemma, we consider $g^{\varepsilon}=g^{\varepsilon}(\xi)$ a measurable function defined on $\varepsilon^{-1} Y^{\prime}$, and another function $\rho=\rho(y ; \eta)$ measurable with respect to $(y ; \eta)$ and $Y$-periodic in $y$. We then introduce

$$
G^{\varepsilon}(x)=\int_{\varepsilon^{-1} Y^{\prime}} g^{\varepsilon}(\xi) e^{i x \cdot \xi} \rho\left(\frac{x}{\varepsilon} ; \varepsilon \xi\right) d \xi, \quad x \in \mathbb{R}^{N} .
$$

The following result estimates its $L^{2}\left(\mathbb{R}^{N}\right)$ and $H^{1}\left(\mathbb{R}^{N}\right)$ norms.

Lemma 2.3 We assume $g^{\varepsilon} \in L^{2}\left(\varepsilon^{-1} Y^{\prime}\right)$ and $\rho \in L^{\infty}\left(Y^{\prime} ; H_{\#}^{1}(Y)\right)$. Then we have

$$
\begin{aligned}
\left\|G^{\varepsilon}\right\|_{L^{2}\left(\mathbb{R}^{N}\right)}^{2} & =\int_{\varepsilon^{-1} Y^{\prime}}\left|g^{\varepsilon}(\xi)\right|^{2}\|\rho(\cdot ; \varepsilon \xi)\|_{L^{2}(Y)}^{2} d \xi \\
\left|G^{\varepsilon}\right|_{H^{1}\left(\mathbb{R}^{N}\right)}^{2} & =\int_{\varepsilon^{-1} Y^{\prime}}\left|g^{\varepsilon}(\xi)\right|^{2}\left\|i \xi \rho(\cdot ; \varepsilon \xi)+\varepsilon^{-1} \nabla_{y} \rho(\cdot ; \varepsilon \xi)\right\|_{L^{2}(Y)^{N}}^{2} d \xi .
\end{aligned}
$$

Proof. We expand $\rho(y ; \eta)$ as a function of $y$ in the orthonormal basis $\left\{\phi_{m}(y ; \eta)\right\}_{m=1}^{\infty}$ where $\eta$ is a parameter:

$$
\rho(y ; \eta)=\sum_{m=1}^{\infty} a_{m}(\eta) \phi_{m}(y ; \eta)
$$

Introducing this expression in (2.2), we get

$$
G^{\varepsilon}(x)=\int_{\varepsilon^{-1} Y^{\prime}} g^{\varepsilon}(\xi) \sum_{m=1}^{\infty} a_{m}(\varepsilon \xi) e^{i x \cdot \xi} \phi_{m}^{\varepsilon}(x ; \xi) d \xi .
$$

Applying the Parseval's identity of Theorem 1.3, we get

$$
\left\|G^{\varepsilon}\right\|_{L^{2}\left(\mathbb{R}^{N}\right)}^{2}=\int_{\varepsilon^{-1} Y^{\prime}}\left|g^{\varepsilon}(\xi)\right|^{2} \sum_{m=1}^{\infty}\left|a_{m}(\varepsilon \xi)\right|^{2} d \xi .
$$

This completes the proof of the first part of the lemma if we use the Parseval's identity in $L^{2}(Y)$ :

$$
\|\rho(\cdot ; \eta)\|_{L^{2}(Y)}^{2}=\sum_{m=1}^{\infty}\left|a_{m}(\eta)\right|^{2} \quad \forall \eta \in Y^{\prime} .
$$


For the second part of the lemma, we differentiate formally $G^{\varepsilon}(x)$ with respect to $x$. We obtain

$$
\nabla_{x} G^{\varepsilon}(x)=\int_{\varepsilon^{-1} Y^{\prime}} g^{\varepsilon}(\xi) e^{i x \cdot \xi}\left(i \xi \rho\left(\frac{x}{\varepsilon} ; \varepsilon \xi\right)+\varepsilon^{-1} \nabla_{y} \rho\left(\frac{x}{\varepsilon} ; \varepsilon \xi\right)\right) d \xi .
$$

We remark that the above integral is of the same type as the one analyzed in the first part. This completes the proof.

The next lemma presents $H^{1}$ estimates on the Bloch modes.

Lemma 2.4 We suppose that the coefficients $a_{k \ell}$ satisfy (1.2). Then there exists a constant $c$, depending on $\left\|a_{k \ell}\right\|_{L^{\infty}(Y)}$ such that

$$
\left\|\frac{\partial \phi_{m}}{\partial y_{k}}(\cdot ; \eta)\right\|_{L^{2}(Y)} \leq c_{1} \lambda_{m}(\eta)^{1 / 2} \quad \forall \eta \in Y^{\prime}, m \geq 1, k=1, \ldots, N .
$$

To prove this, let us introduce the bilinear forms associated with the operators $A(\eta)$ and $A$, respectively.

$$
\begin{aligned}
a(\eta ; \phi, \psi) & =\int_{Y} a_{k \ell}(y)\left(\frac{\partial \phi}{\partial y_{\ell}}+i \eta_{\ell} \phi\right) \overline{\left(\frac{\partial \psi}{\partial y_{k}}+i \eta_{k} \psi\right)} d y, \\
a(\phi, \psi) & =\int_{Y} a_{k \ell}(y) \frac{\partial \phi}{\partial y_{\ell}} \frac{\overline{\partial \psi}}{\partial y_{k}} d y .
\end{aligned}
$$

The basic estimates on them are obtained in [4], p. 190: There exist constants $c, \widetilde{c}$ which are independent of $\eta \in Y^{\prime}$ such that for all $\phi \in H_{\#}^{1}(Y)$

$$
\begin{gathered}
c\left(\|\nabla \phi\|_{L^{2}(Y)^{N}}^{2}+|\eta|^{2}\|\phi\|_{L^{2}(Y)}^{2}\right) \leq a(\eta ; \phi, \phi) \leq \widetilde{c}\left(\|\nabla \phi\|_{L^{2}(Y)^{N}}^{2}+|\eta|^{2}\|\phi\|_{L^{2}(Y)}^{2}\right), \\
c\|\nabla \phi\|_{L^{2}(Y)^{N}}^{2} \leq a(\phi, \phi) \leq \widetilde{c}\|\nabla \phi\|_{L^{2}(Y)^{N}}^{2}
\end{gathered}
$$

Proof of Lemma 2.4 For simplicity, we denote $\phi_{m}(\cdot ; \eta)$ by $\phi_{m}(\eta)$. We recall that it satisfies

$$
a\left(\eta ; \phi_{m}(\eta), \psi\right)=\lambda_{m}(\eta)\left(\phi_{m}(\eta), \psi\right) \quad \forall \psi \in H_{\#}^{1}(Y) .
$$

To deduce (2.4), it is enough to take $\psi=\phi_{m}(\eta)$ and use (2.5).

Our next result concerns the estimation of expressions which are inverse to (2.2). We define

$$
J^{\varepsilon} g(\xi)=\int_{\mathbb{R}^{N}} g(x) e^{-i x \cdot \xi} \rho\left(\frac{x}{\varepsilon} ; \varepsilon \xi\right) d x \quad \text { for } \quad \xi \in \varepsilon^{-1} Y^{\prime},
$$

where $g=g(x)$ is a measurable function defined on $\mathbb{R}^{N}$ and $\rho=\rho(y ; \eta)$ is a measurable function defined on $Y \times Y^{\prime}$. We assume that $\rho$ is $Y$-periodic in $y$. The required hypotheses on these functions will depend on the estimate deduced on $J^{\varepsilon} g$. This is illustrated in the results that follow which are analogous to classical estimates on Fourier transform. 
Lemma $2.5 \quad$ (i) If $g \in L^{2}\left(\mathbb{R}^{N}\right)$ and $\rho \in L^{\infty}\left(Y^{\prime} ; L_{\#}^{2}(Y)\right)$, then we have

$$
\left\|J^{\varepsilon} g\right\|_{L^{2}\left(\varepsilon^{-1} Y^{\prime}\right)} \leq\|g\|_{L^{2}\left(\mathbb{R}^{N}\right)}\|\rho\|_{L^{\infty}\left(Y^{\prime} ; L_{\#}^{2}(Y)\right)} .
$$

(ii) If $g \in H^{1}\left(\mathbb{R}^{N}\right)$ and $\rho \in L^{\infty}\left(Y^{\prime} ; H_{\#}^{1}(Y)\right)$, then we have

$$
\begin{aligned}
\left\|\left(1+|\xi|^{2}\right)^{1 / 2} J^{\varepsilon} g(\xi)\right\|_{L^{2}\left(\varepsilon^{-1} Y^{\prime}\right)} \leq c\{ & \|\nabla g\|_{L^{2}\left(\mathbb{R}^{N}\right)}\|\rho\|_{L^{\infty}\left(Y^{\prime} ; L^{2}(Y)\right)}+ \\
& \left.+\varepsilon^{-1}\|g\|_{L^{2}\left(\mathbb{R}^{N}\right)}\left\|\nabla_{y} \rho\right\|_{L^{\infty}\left(Y^{\prime} ; L^{2}(Y)^{N}\right)}\right\} .
\end{aligned}
$$

Proof. The idea is to consider the product space $L^{2}\left(Y^{\prime} ; L_{\#}^{2}(Y)\right)$ and expand $\rho(y ; \eta)$ in two steps. First using the fact that $\left\{\bar{\phi}_{m}(\cdot ; \eta)\right\}_{m=1}^{\infty}$ is an orthonormal basis in $L_{\#}^{2}(Y)$, we get

$$
\rho(y ; \eta)=\sum_{m=1}^{\infty} a_{m}(\eta) \bar{\phi}_{m}(y ; \eta) \quad \forall y \in Y, \eta \in Y^{\prime}
$$

Next, for each $m$, we can expand $a_{m}(\eta)$ in the usual Fourier series:

$$
a_{m}(\eta)=\sum_{n \in \mathbb{Z}^{N}} a_{m n} e^{2 \pi i n \cdot \eta} \quad \forall \eta \in Y^{\prime}
$$

The corresponding Parseval's identities are as follows:

$$
\begin{aligned}
\|\rho(\cdot ; \eta)\|_{L^{2}(Y)}^{2} & =\sum_{m}\left|a_{m}(\eta)\right|^{2} \quad \forall \eta \in Y^{\prime}, \\
\int_{Y^{\prime}}\left|a_{m}(\eta)\right|^{2} d \eta & =\sum_{n \in \mathbb{Z}^{N}}\left|a_{m n}\right|^{2} \quad \forall m \in \mathbb{N} .
\end{aligned}
$$

Using this expansion, we can rewrite $J^{\varepsilon} g$ as follows:

$$
J^{\varepsilon} g(\xi)=\sum_{m=1}^{\infty} \sum_{n \in \mathbb{Z}^{N}} a_{m n} e^{2 \pi i \varepsilon n \cdot \xi} \int_{\mathbb{R}^{N}} g(x) e^{-i x \cdot \xi} \bar{\phi}_{m}\left(\frac{x}{\varepsilon} ; \varepsilon \xi\right) d x
$$

which, according to the definition of $B_{m}^{\varepsilon} g(\xi)$ is equal to

$$
J^{\varepsilon} g(\xi)=\sum_{m=1}^{\infty} \sum_{n \in \mathbb{Z}^{N}} a_{m n} e^{2 \pi i \varepsilon n \cdot \xi} B_{m}^{\varepsilon} g(\xi)=\sum_{m=1}^{\infty} a_{m}(\varepsilon \xi) B_{m}^{\varepsilon} g(\xi) .
$$

By Cauchy-Schwarz inequality,

$$
\begin{aligned}
\left|J^{\varepsilon} g(\xi)\right|^{2} & \leq\left(\sum_{m=1}^{\infty}\left|a_{m}(\varepsilon \xi)\right|^{2}\right)\left(\sum_{m=1}^{\infty}\left|B_{m}^{\varepsilon} g(\xi)\right|^{2}\right) \\
& =\|\rho(\cdot ; \varepsilon \xi)\|_{L^{2}(Y)}^{2}\left(\sum_{m=1}^{\infty}\left|B_{m}^{\varepsilon} g(\xi)\right|^{2}\right) \\
& \leq\|\rho\|_{L^{\infty}\left(Y^{\prime} ; L_{\#}^{2}(Y)\right)}^{2}\left(\sum_{m=1}^{\infty}\left|B_{m}^{\varepsilon} g(\xi)\right|^{2}\right) .
\end{aligned}
$$


The proof of (i) is complete if we integrate the above inequality with respect to $\xi \in \varepsilon^{-1} Y^{\prime}$ and apply Theorem 1.3. For the proof of (ii), we multiply $(2.8)$ by $\left(-i \xi_{k}\right)$, and obtain

$$
\left(-i \xi_{k}\right) J^{\varepsilon} g(\xi)=\int_{\mathbb{R}^{N}} g(x)\left(-i \xi_{k}\right) e^{-i x \cdot \xi} \rho\left(\frac{x}{\varepsilon} ; \varepsilon \xi\right) d x
$$

which, by integration by parts, can be rewritten as

$$
\left(-i \xi_{k}\right) J^{\varepsilon} g(\xi)=-\int_{\mathbb{R}^{N}} \frac{\partial g}{\partial x_{k}}(x) e^{-i x \cdot \xi} \rho\left(\frac{x}{\varepsilon} ; \varepsilon \xi\right) d x-\varepsilon^{-1} \int_{\mathbb{R}^{N}} g(x) e^{-i x \cdot \xi} \frac{\partial \rho}{\partial y_{k}}\left(\frac{x}{\varepsilon} ; \varepsilon \xi\right) d x .
$$

It is now sufficient to apply $(i)$ to each of the terms on the right hand side of the above relation.

Next, we will need some properties of the classical discrete Fourier transform in our asymptotic description of the first Bloch transform. In particular, we are interested in the relation between discrete and continuous Fourier transforms. To this end, let us begin by introducing some necessary notations. Let $\left\{Y_{\ell}^{\varepsilon}\right\}_{\ell \in \mathbb{Z}^{N}}$ be the mesh of $\mathbb{R}^{N}$ generated by the cell $\varepsilon Y$. More precisely, $Y_{\ell}^{\varepsilon}=x_{\ell}^{\varepsilon}+\varepsilon Y$ where $x_{\ell}^{\varepsilon}=2 \pi \varepsilon \ell$ is the origin of the cell $Y_{\ell}^{\varepsilon}$. We recall the definition of the discrete Fourier transform of a function corresponding to this mesh: Let $p>N$ be given. For $g \in W^{1, p}\left(\mathbb{R}^{N}\right)$ with compact support we define

$$
F^{\varepsilon} g(\xi)=\sum_{\ell \in \mathbb{Z}^{N}} g\left(x_{\ell}^{\varepsilon}\right) e^{-i x_{\ell}^{\varepsilon} \cdot \xi} \quad \forall \xi \in \varepsilon^{-1} Y^{\prime}
$$

It is worthwhile to recall that $W^{1, p}\left(\mathbb{R}^{N}\right)$ is embedded in $\mathcal{C}^{0}\left(\mathbb{R}^{N}\right)$ when $p>N$ and so $g\left(x_{\ell}^{\varepsilon}\right)$ is well-defined.

Lemma 2.6 For $g \in W^{1, p}\left(\mathbb{R}^{N}\right)(p>N)$ with compact support $K$, we have

$$
\text { (i) } \varepsilon^{N}\left(\chi_{\varepsilon^{-1} Y^{\prime}} F^{\varepsilon} g\right)(\xi) \rightarrow \frac{1}{(2 \pi)^{N / 2}} \widehat{g}(\xi) \quad \text { for } \quad \xi \in \mathbb{R}^{N} \text {. }
$$

(ii) $\left\|\varepsilon^{N} F^{\varepsilon} g\right\|_{L^{2}\left(\varepsilon^{-1} Y^{\prime}\right)} \leq c|K|^{\frac{p-2}{2 p}}\left\{\|g\|_{L^{p}\left(\mathbb{R}^{N}\right)}+\varepsilon\|\nabla g\|_{L^{p}\left(\mathbb{R}^{N}\right)^{N}}\right\},|K|$ denotes the measure of $K$.

(iii) $\varepsilon^{N} \chi_{\varepsilon^{-1} Y^{\prime}} F^{\varepsilon} g \rightarrow \frac{1}{(2 \pi)^{N / 2}} \widehat{g} \quad$ in $\quad L^{2}\left(\mathbb{R}^{N}\right)$.

Proof. To prove (i), we multiply $(2.9)$ by $\varepsilon^{N}$ to get

$$
\varepsilon^{N} F^{\varepsilon} g(\xi)=\frac{1}{(2 \pi)^{N}} \sum_{\ell \in \mathbb{Z}^{N}} g\left(x_{\ell}^{\varepsilon}\right) e^{-i x_{\ell}^{\varepsilon} \cdot \xi}\left|Y_{\ell}^{\varepsilon}\right| .
$$

We regard the right side of the above equality as a Riemann sum of the integral

$$
\frac{1}{(2 \pi)^{N}} \int_{\mathbb{R}^{N}} g(x) e^{-i x \cdot \xi} d x
$$

and hence converges to it as $\varepsilon \rightarrow 0$.

To prove (ii), we observe that the right side of (2.9) is nothing but the Fourier series in the variable $\xi \in \varepsilon^{-1} Y^{\prime}$. Therefore, by Parseval's identity, we get

$$
\varepsilon^{N} \int_{\varepsilon^{-1} Y^{\prime}}\left|F^{\varepsilon} g(\xi)\right|^{2} d \xi=\sum_{\ell \in \mathbb{Z}^{N}}\left|g\left(x_{\ell}^{\varepsilon}\right)\right|^{2} .
$$


We multiply this relation by $\varepsilon^{N}$ and rewrite it as

$$
\varepsilon^{2 N} \int_{\varepsilon^{-1} Y^{\prime}}\left|F^{\varepsilon} g(\xi)\right|^{2} d \xi=\frac{1}{(2 \pi)^{N}} \sum_{\ell \in \mathbb{Z}^{N}}\left|g\left(x_{\ell}^{\varepsilon}\right)\right|^{2}\left|Y_{\ell}^{\varepsilon}\right| .
$$

To estimate the right side of the above equality, we integrate the inequality

$$
\left|g\left(x_{\ell}^{\varepsilon}\right)\right|^{2} \leq 2\left\{|g(x)|^{2}+\left|g(x)-g\left(x_{\ell}^{\varepsilon}\right)\right|^{2}\right\}, \quad x \in Y_{\ell}^{\varepsilon} .
$$

over $Y_{\ell}^{\varepsilon}$ to obtain

$$
\left|g\left(x_{\ell}^{\varepsilon}\right)\right|^{2}\left|Y_{\ell}^{\varepsilon}\right| \leq 2\left\{\int_{Y_{\ell}^{\varepsilon}}|g(x)|^{2} d x+\int_{Y_{\ell}^{\varepsilon}}\left|g(x)-g\left(x_{\ell}^{\varepsilon}\right)\right|^{2} d x\right\} .
$$

Since $p>N$, we can use the classical Morrey's inequality (see BrEzIs [2], p. 167) to deduce

$$
\left|g(x)-g\left(x_{\ell}^{\varepsilon}\right)\right| \leq c \varepsilon^{1-\frac{N}{p}}|\nabla g|_{L^{p}\left(Y_{\ell}^{\varepsilon}\right)^{N}} .
$$

Using the above estimate in (2.11) and using Hölder inequality, and summing over $\ell \in \mathbb{Z}^{N}$, we complete the proof of (ii).

The statement (iii) follows from the arguments based on Egorov's Theorem because (ii) implies weak convergence in $L^{2}\left(\mathbb{R}^{N}\right)$ of the sequence under consideration and (i) implies its point-wise convergence.

\section{$3 \quad$ Higher Bloch modes are negligible}

In this section, we consider a sequence of solutions $u^{\varepsilon}$ of the equation with $f \in H^{-1}\left(\mathbb{R}^{N}\right)$ :

$$
A^{\varepsilon} u^{\varepsilon}=f \quad \text { in } \quad \mathbb{R}^{N}, \quad u^{\varepsilon} \in H^{1}\left(\mathbb{R}^{N}\right) .
$$

Let us recall that the above equation is equivalent to (1.20) in the Bloch space. In what follows, we present a systematic method of obtaining estimates on the solution in Sobolev spaces $L^{2}$ and $H^{1}$. In particular, we show that the component of $u^{\varepsilon}$ in the higher Bloch modes do not play any role in the analysis of correctors of first and second order provided $f$ is sufficiently smooth. Thus we consider $v^{\varepsilon}$ defined in (1.21) which is nothing but the projection of $u^{\varepsilon}$ corresponding to all higher Bloch modes. Estimates on $v^{\varepsilon}$ derived in this section improve Proposition 1.4.

Proposition 3.1 We have the following estimates for $f \in L^{2}\left(\mathbb{R}^{N}\right)$,

(i) $\quad\left|v^{\varepsilon}\right|_{H^{1}\left(\mathbb{R}^{N}\right)} \leq c \varepsilon\|f\|_{L^{2}\left(\mathbb{R}^{N}\right)}$,

(ii) $\quad\left\|v^{\varepsilon}\right\|_{L^{2}\left(\mathbb{R}^{N}\right)} \leq c \varepsilon\|f\|_{H^{-1}\left(\mathbb{R}^{N}\right)}$. 
Proof. To show (i), we apply Lemma 2.1 with $g=v^{\varepsilon}$, and use the equation (1.20). We obtain

$$
\begin{aligned}
\left\|\nabla v^{\varepsilon}\right\|_{L^{2}\left(\mathbb{R}^{N}\right)^{N}}^{2} & \leq c \int_{\varepsilon^{-1} Y^{\prime}} \sum_{m=2}^{\infty} \frac{1}{\lambda_{m}^{\varepsilon}(\xi)}\left|B_{m}^{\varepsilon} f(\xi)\right|^{2} d \xi \\
& \leq c \sup _{m \geq 2, \xi \in \varepsilon^{-1} Y^{\prime}} \frac{1}{\lambda_{m}^{\varepsilon}(\xi)}\|f\|_{L^{2}\left(\mathbb{R}^{N}\right)}^{2} .
\end{aligned}
$$

Proof of (i) is over since we have (cf. (1.25)):

$$
\sup _{m \geq 2,} \frac{1}{\xi \in \varepsilon^{-1} Y^{\prime}}, \frac{1}{\lambda_{m}^{\varepsilon}(\xi)} \varepsilon_{2}^{2} .
$$

For the proof of (ii), we apply Lemma 2.2 with $g=f$ and the equation (1.20). We have

$$
\begin{aligned}
\left\|v^{\varepsilon}\right\|_{L^{2}\left(\mathbb{R}^{N}\right)}^{2} & =\int_{\varepsilon^{-1} Y^{\prime}} \sum_{m=2}^{\infty}\left|B_{m}^{\varepsilon} u^{\varepsilon}(\xi)\right|^{2} d \xi \\
& =\int_{\varepsilon^{-1} Y^{\prime}} \sum_{m=2}^{\infty} \frac{1}{\lambda_{m}^{\varepsilon}(\xi)^{2}}\left|B_{m}^{\varepsilon} f(\xi)\right|^{2} d \xi .
\end{aligned}
$$

Writing

$$
\frac{1}{\lambda_{m}^{\varepsilon}(\xi)^{2}}\left|B_{m}^{\varepsilon} f(\xi)\right|^{2}=\frac{1+\lambda_{m}^{\varepsilon}(\xi)}{\lambda_{m}^{\varepsilon}(\xi)^{2}} \frac{\left|B_{m}^{\varepsilon} f(\xi)\right|^{2}}{1+\lambda_{m}^{\varepsilon}(\xi)}
$$

and using (3.2), we deduce that

$$
\frac{1}{\lambda_{m}^{\varepsilon}(\xi)^{2}}\left|B_{m}^{\varepsilon} f(\xi)\right|^{2} \leq c \varepsilon^{2} \frac{\left|B_{m}^{\varepsilon} f(\xi)\right|^{2}}{1+\lambda_{m}^{\varepsilon}(\xi)}
$$

The proof is complete if we use Lemma 2.2.

While the above proposition shows that $v^{\varepsilon}$ can be neglected at the level of the first order correctors (cf. (1.10)), the next result will demonstrate that $v^{\varepsilon}$ can be neglected at the level of correctors of first and second order. These finer estimates require naturally higher order regularity of $f$ but not of the coefficients $a_{k \ell}(y)$. Let us state the following proposition whose proof is similar to the previous one and hence will not be repeated.

Proposition 3.2 We have the following estimates for $f \in H^{1}\left(\mathbb{R}^{N}\right)$,

(i) $\quad\left|v^{\varepsilon}\right|_{H^{1}\left(\mathbb{R}^{N}\right)} \leq c \varepsilon^{2}\|f\|_{H^{1}\left(\mathbb{R}^{N}\right)}$,

$$
\left\|v^{\varepsilon}\right\|_{L^{2}\left(\mathbb{R}^{N}\right)} \leq c \varepsilon^{2}\|f\|_{L^{2}\left(\mathbb{R}^{N}\right)} .
$$

Assuming $a_{k \ell} \in W_{\#}^{1, \infty}(Y)$, we can obtain $H^{2}$-estimates on the solution. This is difficult as it involves more subtleties (see [6]). 


\section{Taylor expansion of the first Bloch eigenvalue and eigenvector}

The purpose of this section is to indicate a systematic method to compute derivatives of the first Bloch eigenvalue $\lambda_{1}(\eta)$ and the first Bloch eigenvector $\phi_{1}(\cdot ; \eta)$ at $\eta=0$. In particular, we will be proving Proposition 1.9 and 1.10. Recall that $\lambda_{1}(\eta)$ and $\phi_{1}(\cdot ; \eta)$ depend analytically on $\eta$ in a small neighbourhood $B_{\delta}$ of $\eta=0$. At the cost of reducing this neighbourhood, we claim that the branch $\eta \mapsto \phi_{1}(\cdot ; \eta)$ can be so chosen that the following conditions are satisfied simultaneously:

$$
\begin{aligned}
\eta \in B_{\delta} \mapsto \phi_{1}(\cdot ; \eta) \in H_{\#}^{1}(Y) & \text { is analytic, } \\
\left\|\phi_{1}(\cdot ; \eta)\right\|_{L^{2}(Y)}=1 & \forall \eta \in B_{\delta}, \\
\mathfrak{I m}_{\int_{Y}} \phi_{1}(y ; \eta) d y=0 & \forall \eta \in B_{\delta} .
\end{aligned}
$$

In the sequel, we will see that the above conditions fix uniquely the eigenvector $\phi_{1}(\cdot ; \eta)$. We remark that the condition (4.2) is classical whereas the condition (4.3) is somewhat unusual and it can be achieved as indicated below. The idea consists of multiplying $\phi_{1}(\cdot ; \eta)$ by a complex number $\left(\alpha_{1}(\eta)+i \alpha_{2}(\eta)\right)$ where $\alpha_{1}(\eta)$ and $\alpha_{2}(\eta)$ are real analytic with respect to $\eta$ and are chosen such that

$$
\mathfrak{I m} \int_{Y}\left(\alpha_{1}(\eta)+i \alpha_{2}(\eta)\right) \phi_{1}(y ; \eta) d y=0 .
$$

If we define

$$
d(\eta)=\left(d_{1}(\eta), d_{2}(\eta)\right) \stackrel{\text { def }}{=}\left(\mathfrak{I m} \int_{Y} \phi_{1}(y ; \eta) d y, \mathfrak{R e} \int_{Y} \phi_{1}(y ; \eta) d y\right)
$$

then the above condition is equivalent to

$$
\alpha_{1}(\eta) d_{1}(\eta)+\alpha_{2}(\eta) d_{2}(\eta)=0 \quad \forall \eta \in B_{\delta} .
$$

Obviously, one such choice which is analytic is as follows:

$$
\alpha_{1}(\eta)=-d_{2}(\eta), \quad \alpha_{2}(\eta)=d_{1}(\eta) .
$$

Of course, the above procedure has destroyed the condition (4.2) (but not condition (4.1)). However, it can be regained by dividing by $|d(\eta)|$. This is possible because $d(0) \neq 0$ by our choice of $\phi_{1}(\cdot ; 0)$ (see Proposition 1.5).

Thanks to our choice of the branch satisfying (4.1) - (4.3), we will now draw some consequences which will simplify the computations below. In fact, differentiating (4.2) with respect to $\eta$, we get successively for all $k, \ell, m, n=1, \ldots, N$,

$$
\begin{gathered}
\mathfrak{R e}\left\langle D_{k} \phi_{1}(\cdot ; \eta), \phi_{1}(\cdot ; \eta)\right\rangle=0, \\
\mathfrak{R e}\left\langle D_{k \ell}^{2} \phi_{1}(\cdot ; \eta), \phi_{1}(\cdot ; \eta)\right\rangle+\mathfrak{R e}\left\langle D_{k} \phi_{1}(\cdot ; \eta), D_{\ell} \phi_{1}(\cdot ; \eta)\right\rangle=0, \\
\left\{\begin{array}{c}
\mathfrak{R e}\left\langle D_{k \ell m}^{3} \phi_{1}(\cdot ; \eta), \phi_{1}(\cdot ; \eta)\right\rangle+\mathfrak{R e}\left\langle D_{k \ell}^{2} \phi_{1}(\cdot ; \eta), D_{m} \phi_{1}(\cdot ; \eta)\right\rangle+ \\
+\mathfrak{R e}\left\langle D_{k m}^{2} \phi_{1}(\cdot ; \eta), D_{\ell} \phi_{1}(\cdot ; \eta)\right\rangle+\mathfrak{R e}\left\langle D_{k} \phi_{1}(\cdot ; \eta), D_{\ell m}^{2} \phi_{1}(\cdot ; \eta)\right\rangle=0,
\end{array}\right.
\end{gathered}
$$




$$
\left\{\begin{array}{c}
\mathfrak{R e}\left\langle D_{k \ell m n}^{4} \phi_{1}(\cdot ; \eta), \phi_{1}(\cdot ; \eta)\right\rangle+\mathfrak{R e}\left\langle D_{k \ell m}^{3} \phi_{1}(\cdot ; \eta), D_{n} \phi_{1}(\cdot ; \eta)\right\rangle+ \\
+\operatorname{Re}\left\langle D_{k \ell n}^{3} \phi_{1}(\cdot ; \eta), D_{m} \phi_{1}(\cdot ; \eta)\right\rangle+\mathfrak{R e}\left\langle D_{k \ell}^{2} \phi_{1}(\cdot ; \eta), D_{m n}^{2} \phi_{1}(\cdot ; \eta)\right\rangle+ \\
+\operatorname{Re}\left\langle D_{k m n}^{3} \phi_{1}(\cdot ; \eta), D_{\ell} \phi_{1}(\cdot ; \eta)\right\rangle+\mathfrak{R e}\left\langle D_{k m}^{2} \phi_{1}(\cdot ; \eta), D_{\ell n}^{2} \phi_{1}(\cdot ; \eta)\right\rangle+ \\
+\mathfrak{R e}\left\langle D_{k n}^{2} \phi_{1}(\cdot ; \eta), D_{\ell m}^{2} \phi_{1}(\cdot ; \eta)\right\rangle+\mathfrak{R e}\left\langle D_{k} \phi_{1}(\cdot ; \eta), D_{\ell m n}^{3} \phi_{1}(\cdot ; \eta)\right\rangle=0,
\end{array}\right.
$$

where $\langle\cdot ; \cdot\rangle$ denotes the scalar product in $L_{\#}^{2}(Y)$. On the other hand, differentiation of (4.3) yields

$$
\mathfrak{I m} \int_{Y} D^{\beta} \phi_{1}(y ; \eta) d y=0 \quad \text { for all } \beta \in \mathbb{Z}_{+}^{N}
$$

From these sets of relations, it follows that

$$
\int_{Y} D^{\beta} \phi_{1}(y ; 0) d y=0 \text { for all } \beta \in \mathbb{Z}_{+}^{N} \text { with }|\beta| \text { odd. }
$$

\subsection{First order derivatives}

If we differentiate the eigenvalue equation $\left(A(\eta)-\lambda_{1}(\eta)\right) \phi_{1}(\cdot ; \eta)=0$, once with respect to $\eta_{k}$, we obtain

$$
D_{k}\left(A(\eta)-\lambda_{1}(\eta)\right) \phi_{1}(\cdot ; \eta)+\left(A(\eta)-\lambda_{1}(\eta)\right) D_{k} \phi_{1}(\cdot ; \eta)=0
$$

Taking scalar product with $\phi_{1}(\cdot ; \eta)$, we get

$$
\left\langle\left[D_{k}\left(A-\lambda_{1}\right)\right] \phi_{1}, \phi_{1}\right\rangle=0,
$$

where we have suppressed the dependence on $\eta$ for ease of writing. We will continue with this convention in the sequel provided there is no ambiguity. It follows from (1.18) that

$$
D_{k} A(0)=i C_{k} \quad \forall \eta \in Y^{\prime}
$$

where the operator $C_{k}$ is defined in (1.19).

If we evaluate the relation (4.11) at $\eta=0$ and use the structure of $C_{k}$, we get immediately that

$$
D_{k} \lambda_{1}(0)=0 \quad \forall k=1, \ldots, N .
$$

The next step is to compute the first order derivatives of $\phi_{1}$ at $\eta=0$. To this end, we go back to (4.10) and use (4.13). We obtain

$$
A D_{k} \phi_{1}(\cdot ; 0)=-D_{k} A(0) \phi_{1}(\cdot ; 0)=-i C_{k} \phi_{1}(\cdot ; 0) .
$$

Taking into account (4.9) and the above equation, we can solve uniquely for $D_{k} \phi_{1}(y ; 0)$, and obtain

$$
D_{k} \phi_{1}(y ; 0)=i \phi_{1}(y ; 0) \chi_{k}(y)=i p^{(0)} \chi_{k}(y)
$$

where, we recall, $\chi_{k}$ satisfies (1.13) and the constant $p^{(0)}$ was fixed in Proposition 1.5. Thus, the first order derivative is completely determined and

$$
D_{k} \phi_{1}(y ; 0) \text { is purely imaginary. }
$$




\subsection{Second order derivatives}

Our starting point is the relation (4.10) which we differentiate once with respect to $\eta$. We obtain

$$
\left[D_{k \ell}^{2}\left(A-\lambda_{1}\right)\right] \phi_{1}+\left[D_{k}\left(A-\lambda_{1}\right)\right] D_{\ell} \phi_{1}+\left[D_{\ell}\left(A-\lambda_{1}\right)\right] D_{k} \phi_{1}+\left(A-\lambda_{1}\right) D_{k \ell}^{2} \phi_{1}=0 .
$$

Taking scalar product with $\phi_{1}$, we get

$$
\left\langle\left[D_{k \ell}^{2}\left(A-\lambda_{1}\right)\right] \phi_{1}, \phi_{1}\right\rangle+\left\langle\left[D_{k}\left(A-\lambda_{1}\right)\right] D_{\ell} \phi_{1}, \phi_{1}\right\rangle+\left\langle\left[D_{\ell}\left(A-\lambda_{1}\right)\right] D_{k} \phi_{1}, \phi_{1}\right\rangle=0,
$$

for all $\eta \in B_{\delta}$. If we use the information obtained in Paragraph 4.1 on $D_{k} \lambda_{1}(0), D_{k} \phi_{1}(\cdot ; 0), D_{k} A(0)$, and the following

$$
D_{k \ell}^{2} A(\eta)=2 a_{k \ell}(y) \quad \forall k, \ell=1, \ldots, N, \eta \in Y^{\prime},
$$

we obtain

$$
\begin{aligned}
\frac{1}{2 !} D_{k \ell}^{2} \lambda_{1}(0) & =\frac{1}{|Y|} \int_{Y} a_{k \ell}(y) d y-\frac{1}{2|Y|} \int_{Y}\left(C_{k} \chi_{\ell}(y)+C_{\ell} \chi_{k}(y)\right) d y \\
& =\frac{1}{2}\left(q_{k \ell}+q_{\ell k}\right)=q_{k \ell} \quad \forall k, \ell=1, \ldots, N .
\end{aligned}
$$

As before, the next step is to compute $D_{k \ell}^{2} \phi_{1}(\cdot ; 0)$. For this purpose, we go back to (4.16) and rewrite it with $\eta=0$ as follows:

$$
A D_{k \ell}^{2} \phi_{1}(\cdot ; 0)=\left\{-2\left(a_{k \ell}-q_{k \ell}\right)+C_{k} \chi_{\ell}+C_{\ell} \chi_{k}\right\} \phi_{1}(\cdot ; 0) .
$$

By comparing the above equation with (1.30) and using the simplicity of the eigenvalue under consideration, we see that $D_{k \ell}^{2} \phi_{1}(\cdot ; 0)$ is of the form

$$
\frac{1}{2 !} D_{k \ell}^{2} \phi_{1}(y ; 0)=-p^{(0)} \chi_{k \ell}(y)-\beta_{k \ell}^{(2)} p^{(0)}
$$

for some constant $\beta_{k \ell}^{(2)}$. Thanks to (4.5) and (4.8), we can infer that

$$
\beta_{k \ell}^{(2)} \text { and } D_{k \ell}^{2} \phi_{1}(\cdot ; 0) \text { are real. }
$$

Moreover, $\beta_{k \ell}^{(2)}$ admits the expression given in Proposition 1.10.

\subsection{Third order derivatives}

From the calculations done so far, it is now clear how to proceed further to calculate higher order derivatives. So, we will be brief here. Differentiating (4.16), we get

$$
\left\{\begin{array}{c}
{\left[D_{k \ell m}^{3}\left(A-\lambda_{1}\right)\right] \phi_{1}+\left[D_{k \ell}^{2}\left(A-\lambda_{1}\right)\right] D_{m} \phi_{1}+\left[D_{\ell m}^{2}\left(A-\lambda_{1}\right)\right] D_{k} \phi_{1}+} \\
\quad+\left[D_{k m}^{2}\left(A-\lambda_{1}\right)\right] D_{\ell} \phi_{1}+\left[D_{k}\left(A-\lambda_{1}\right)\right] D_{\ell m}^{2} \phi_{1}+\left[D_{\ell}\left(A-\lambda_{1}\right)\right] D_{k m}^{2} \phi_{1}+ \\
\quad+\left[D_{m}\left(A-\lambda_{1}\right)\right] D_{k \ell}^{2} \phi_{1}+\left(A-\lambda_{1}\right) D_{k \ell m}^{3} \phi_{1}=0
\end{array}\right.
$$

Taking scalar product with $\phi_{1}$, we get

$$
\left\{\begin{array}{c}
\left\langle\left[D_{k \ell m}^{3}\left(A-\lambda_{1}\right)\right] \phi_{1}, \phi_{1}\right\rangle+\left\langle\left[D_{k \ell}^{2}\left(A-\lambda_{1}\right)\right] D_{m} \phi_{1}, \phi_{1}+\left\langle\left[D_{\ell m}^{2}\left(A-\lambda_{1}\right)\right] D_{k} \phi_{1}, \phi_{1}\right\rangle+\right. \\
+\left\langle\left[D_{k m}^{2}\left(A-\lambda_{1}\right)\right] D_{\ell} \phi_{1}, \phi_{1}\right\rangle+\left\langle\left[D_{k}\left(A-\lambda_{1}\right)\right] D_{\ell m}^{2} \phi_{1}, \phi_{1}\right\rangle+ \\
+\left\langle\left[D_{\ell}\left(A-\lambda_{1}\right)\right] D_{k m}^{2} \phi_{1}, \phi_{1}\right\rangle+\left\langle\left[D_{m}\left(A-\lambda_{1}\right)\right] D_{k \ell}^{2} \phi_{1}, \phi_{1}\right\rangle=0
\end{array}\right.
$$


To conclude that $D_{k \ell m}^{3} \lambda_{1}(0)=0$, it is enough to use the following information in the above relation:

$$
\left\{\begin{array}{l}
D_{k} A \text { is purely imaginary, } \quad D_{k \ell}^{2} A \text { is real, } \quad D_{k \ell m}^{3} A=0, \\
\phi_{1}(0), D_{k \ell}^{2} \phi_{1}(0) \text { are real, } \quad D_{k} \phi_{1}(0) \text { is purely imaginary. }
\end{array}\right.
$$

It is evident that the above argument is very general and so can be used to establish that all odd order derivatives of $\lambda_{1}$ at $\eta=0$ vanish. This proves the first part of Proposition 1.9.

To find the third order derivatives of $\phi_{1}$ at $\eta=0$, we realize that (4.21) defines a periodic problem for $D_{k \ell m}^{3} \phi_{1}(\cdot ; 0)$ which can be compared with (1.31). Further, the relation (4.9) says that its average vanishes. These observations are enough to get the expression of $D_{k \ell m}^{3} \phi_{1}(\cdot ; 0)$ given in Proposition 1.10. We conclude by observing the following important property:

$$
D_{k \ell m}^{3} \phi_{1}(y ; 0) \text { is purely imaginary. }
$$

\subsection{Fourth order derivatives}

To arrive at the expressions for the fourth order derivatives of $\lambda_{1}$ and $\phi_{1}$ at $\eta=0$ given in Propositions 1.9 and 1.10, we follow the same arguments as in Paragraph 4.3.

\section{Convergence of the first Bloch transform to Fourier transform}

This section is devoted to the proof of next proposition which shows the sense in which Fourier transform is approximated by the first Bloch transform.

\section{Proposition 5.1}

(i) For every $g \in L^{2}\left(\mathbb{R}^{N}\right)$ with compact support, we have

$$
\chi_{\varepsilon^{-1} Y^{\prime}}(\xi) B_{1}^{\varepsilon} g(\xi) \rightarrow \widehat{g}(\xi) \quad \text { in } \quad L_{l o c}^{\infty}\left(\mathbb{R}_{\xi}^{N}\right) .
$$

(ii) If $g \in L^{2}\left(\mathbb{R}^{N}\right)$, we have

$$
\chi_{\varepsilon^{-1} Y^{\prime}}(\xi) B_{1}^{\varepsilon} g(\xi) \rightarrow \widehat{g}(\xi) \quad \text { in } \quad L^{2}\left(\mathbb{R}_{\xi}^{N}\right) .
$$

This will be a consequence of a more general result. In order to state it, we need to introduce some new notations. To every function $\rho=\rho(y ; \eta)$ defined on $Y \times Y^{\prime}$ which is $Y$-periodic in $y$, we associate the following function:

$$
\widetilde{\rho}^{(0)}(\eta)=\frac{1}{|Y|} \int_{Y} \rho(y ; \eta) e^{-i y \cdot \eta} d y, \quad \eta \in Y^{\prime} .
$$

With this notation, we have

Proposition 5.2 We suppose $\rho \in L^{\infty}\left(Y^{\prime} ; L_{\#}^{2}(Y)\right)$. Then for all $g \in W^{1, p}\left(\mathbb{R}^{N}\right)$ with compact support $K$ and with $p>N$, we have

$$
\chi_{\varepsilon^{-1} Y^{\prime}}(\xi)\left(J^{\varepsilon} g(\xi)-(2 \pi)^{N / 2} \widetilde{\rho}^{(0)}(\varepsilon \xi) \widehat{g}(\xi)\right) \rightarrow 0 \quad \text { in } \quad L^{2}\left(\mathbb{R}_{\xi}^{N}\right) .
$$

where, we recall, $J^{\varepsilon} g$ was defined in (2.8).

The proof will be taken up later. Admitting it for the moment, we turn our attention to 
Proof of Proposition 5.1 If $g \in L^{2}\left(\mathbb{R}^{N}\right)$ with compact support $K$, we have for all $\xi \in \mathbb{R}^{N}$ :

$$
\begin{aligned}
\left|\chi_{\varepsilon^{-1} Y^{\prime}}(\xi) B_{1}^{\varepsilon} g(\xi)-\widehat{g}(\xi)\right| & \leq\left|\chi_{\varepsilon^{-1} Y^{\prime}}(\xi)\left(B_{1}^{\varepsilon} g(\xi)-\widehat{g}(\xi)\right)\right|+\left|\left(\chi_{\varepsilon^{-1} Y^{\prime}}(\xi)-1\right) \widehat{g}(\xi)\right| \\
& \leq c|K|\|g\|_{L^{2}\left(\mathbb{R}^{N}\right)}\left\|\phi_{1}(\cdot ; \varepsilon \xi)-\phi_{1}(\cdot ; 0)\right\|_{L^{2}(Y)}+\left|\left(\chi_{\varepsilon^{-1} Y^{\prime}}(\xi)-1\right) \widehat{g}(\xi)\right| .
\end{aligned}
$$

If $|\xi|$ is bounded, then using the fact that the map $\eta \mapsto \phi_{1}(\cdot ; \eta) \in L_{\#}^{2}(Y)$ is Lipschitz near $\eta=0$, we deduce

$$
\left\|\phi_{1}(\cdot ; \varepsilon \xi)-\phi_{1}(\cdot ; 0)\right\|_{L^{2}(Y)} \leq c \varepsilon
$$

This completes the proof of (i).

The proof of (ii) is more involved. Firstly, according to Theorem 1.3, we have the uniform estimate:

$$
\int_{\varepsilon^{-1} Y^{\prime}}\left|B_{1}^{\varepsilon} g(\xi)\right|^{2} d \xi \leq \int_{\mathbb{R}^{N}}|g(x)|^{2} d x
$$

and so, by the usual density arguments, it is enough to prove (ii) with $g \in \mathcal{D}\left(\mathbb{R}^{N}\right)$. We can now complete the proof using Proposition 5.2. Indeed, with $\rho=\phi_{1}$, we see that

$$
\tilde{\rho}^{(0)}(\varepsilon \xi) \rightarrow p^{(0)} \quad \text { and } \quad B_{1}^{\varepsilon} g(\xi)=J^{\varepsilon} g(\xi) \quad \forall \xi \in \mathbb{R}^{N},
$$

which implies, by Lebesgue's dominated convergence theorem, that

$$
(2 \pi)^{N / 2} \chi_{\varepsilon^{-1} Y^{\prime}}(\xi) \tilde{\rho}^{(0)}(\varepsilon \xi) \widehat{g}(\xi) \rightarrow \widehat{g}(\xi) \quad \text { in } \quad L^{2}\left(\mathbb{R}_{\xi}^{N}\right) .
$$

Proof of Proposition 5.2 The key point is that the variation of $\rho\left(\frac{x}{\varepsilon} ; \varepsilon \xi\right)$ with respect to $x$ is faster than that of $g$. To exploit this, we consider the $\varepsilon$-mesh $\left\{Y_{\ell}^{\varepsilon}\right\}_{\ell \in \mathbb{Z}^{N}}$ generated by the cell $\varepsilon Y$ which was already introduced at the end of Section 2. We decompose

$$
J^{\varepsilon} g(\xi)=\sum_{\ell \in \mathbb{Z}^{N} Y_{\ell}^{\varepsilon}} g(x) e^{-i x \cdot \xi} \rho\left(\frac{x}{\varepsilon} ; \varepsilon \xi\right) d x=\sum_{\ell \in \mathbb{Z}^{N}} g\left(x_{\ell}^{\varepsilon}\right) \int_{Y_{\ell}^{\varepsilon}} e^{-i x \cdot \xi} \rho\left(\frac{x}{\varepsilon} ; \varepsilon \xi\right) d x+r_{1}^{\varepsilon}(\xi),
$$

where

$$
r_{1}^{\varepsilon}(\xi)=\sum_{\ell \in \mathbb{Z}^{N}} \int_{Y_{\ell}^{\varepsilon}}\left(g(x)-g\left(x_{\ell}^{\varepsilon}\right)\right) e^{-i x \cdot \xi} \rho\left(\frac{x}{\varepsilon} ; \varepsilon \xi\right) d x
$$

The first term on the right side of (5.3) can be, by means of the change of variables $x=x_{\ell}^{\varepsilon}+\varepsilon y$, transformed into

$$
|Y| \varepsilon^{N} F^{\varepsilon} g(\xi) \widetilde{\rho}^{(0)}(\varepsilon \xi)
$$

where $F^{\varepsilon} g$ is the discrete Fourier transform of $g$ and $\widetilde{\rho}^{(0)}$ is defined in (5.1). Since we know that $\chi_{\varepsilon^{-1} Y^{\prime}}(\xi) \varepsilon^{N} F^{\varepsilon} g(\xi) \rightarrow \frac{1}{(2 \pi)^{N / 2}} \hat{g}(\xi)$ in $L^{2}\left(\mathbb{R}^{N}\right)$ (cf. Lemma 2.6), our hypothesis on $\rho$ ensures that

$$
\left\|\chi_{\varepsilon^{-1} Y^{\prime}}(\xi)\left\{|Y| \varepsilon^{N} F^{\varepsilon} g(\xi)-(2 \pi)^{N / 2} \widehat{g}(\xi)\right\} \widetilde{\rho}^{(0)}(\varepsilon \xi)\right\|_{L^{2}\left(\mathbb{R}^{N}\right)} \rightarrow 0 .
$$


Thus, to complete the proof, it is enough to show that

$$
\left\|r_{1}^{\varepsilon}\right\|_{L^{2}\left(\varepsilon^{-1} Y^{\prime}\right)} \leq \frac{c(K)}{\left(1-\frac{N}{p}\right)} \varepsilon\|\rho\|_{L^{\infty}\left(Y^{\prime} ; L_{\#}^{2}(Y)\right)}\|\nabla g\|_{L^{p}\left(\mathbb{R}^{N}\right)} .
$$

To this end, we rewrite $r_{1}^{\varepsilon}$ in a slightly different form, namely

$$
r_{1}^{\varepsilon}(\xi)=\int_{\mathbb{R}^{N}} \widetilde{g}_{1}^{\varepsilon}(x) e^{-i x \cdot \xi} \rho\left(\frac{x}{\varepsilon} ; \varepsilon \xi\right) d x,
$$

where

$$
\widetilde{g}_{1}^{\varepsilon}(x)=\sum_{\ell \in \mathbb{Z}^{N}}\left(g(x)-g\left(x_{\ell}^{\varepsilon}\right)\right) \chi_{Y_{\ell}^{\varepsilon}}(x) .
$$

We already know how to estimate integrals of the type (5.7) in $L^{2}\left(\mathbb{R}^{N}\right)$ (see Lemma 2.5) and so we can deduce (5.6) provided we have the estimate

$$
\left\|\widetilde{g}_{1}^{\varepsilon}\right\|_{L^{2}\left(\mathbb{R}^{N}\right)} \leq \frac{c(K)}{\left(1-\frac{N}{p}\right)} \varepsilon\|\nabla g\|_{L^{p}\left(\mathbb{R}^{N}\right)} .
$$

Thanks to our hypothesis, we can deduce a stronger estimate, namely

$$
\left\|\widetilde{g}_{1}^{\varepsilon}\right\|_{L^{p}\left(\mathbb{R}^{N}\right)} \leq \frac{c}{\left(1-\frac{N}{p}\right)} \varepsilon\|\nabla g\|_{L^{p}\left(\mathbb{R}^{N}\right)},
$$

where $c$ is a constant independent of $K$, the support of $g$. We note that (5.10) is a simple consequence of Morrey's estimate (see [2], p. 167).

Finally, we note that (5.9) can be obtained from (5.10) with $c(K)=c|K|^{1-\frac{2}{p}}$ and a simple application of Hölder inequality.

The proof of Proposition 5.2 shows that the result can be strengthened by assuming suitable smoothness on $g$. Our next result is an example in this direction. It introduces naturally the following quantities:

$$
\widetilde{\rho}^{(k)}(\eta)=\frac{1}{|Y|} \int_{Y} \rho(y ; \eta) y_{k} e^{-i y \cdot \eta} d y \quad \forall k=1, \ldots, N, \quad \eta \in Y^{\prime} .
$$

Then we have the following corrector result for $J^{\varepsilon} g$ :

Proposition 5.3 We suppose $\rho \in L^{\infty}\left(Y^{\prime} ; L_{\#}^{2}(Y)\right)$. Then for all $g \in W^{2, p}\left(\mathbb{R}^{N}\right)$ with compact support $K$ and with $p>N$, we have

$$
\chi_{\varepsilon^{-1} Y^{\prime}}(\xi)\left\{J^{\varepsilon} g(\xi)-(2 \pi)^{N / 2}\left[\widetilde{\rho}^{(0)}(\varepsilon \xi)+i \varepsilon \xi_{k} \widetilde{\rho}^{(k)}(\varepsilon \xi)\right] \widehat{g}(\xi)\right\} \rightarrow 0 \quad \text { in } \quad L^{2}\left(\mathbb{R}_{\xi}^{N}\right) .
$$


Proof. We follow the idea of the proof of Proposition 5.2. We decompose $J^{\varepsilon} g(\xi)$ as

$$
J^{\varepsilon} g(\xi)=\sum_{\ell \in \mathbb{Z}^{N}} \int_{Y_{\ell}^{\varepsilon}}\left\{g\left(x_{\ell}^{\varepsilon}\right)+\nabla g\left(x_{\ell}^{\varepsilon}\right) \cdot\left(x-x_{\ell}^{\varepsilon}\right)\right\} e^{-i x \cdot \xi} \rho\left(\frac{x}{\varepsilon} ; \varepsilon \xi\right) d x+r_{2}^{\varepsilon}(\xi),
$$

where

$$
r_{2}^{\varepsilon}(\xi)=\sum_{\ell \in \mathbb{Z}^{N}} \int_{Y_{\ell}^{\varepsilon}}\left\{g(x)-g\left(x_{\ell}^{\varepsilon}\right)-\nabla g\left(x_{\ell}^{\varepsilon}\right) \cdot\left(x-x_{\ell}^{\varepsilon}\right)\right\} e^{-i x \cdot \xi} \rho\left(\frac{x}{\varepsilon} ; \varepsilon \xi\right) d x
$$

We can estimate $r_{2}^{\varepsilon}(\xi)$ as follows:

$$
\left\|r_{2}^{\varepsilon}\right\|_{L^{2}\left(\varepsilon^{-1} Y^{\prime}\right)} \leq \frac{c(K)}{\left(2-\frac{N}{p}\right)} \varepsilon^{2}\|\rho\|_{L^{\infty}\left(Y^{\prime} ; L_{\#}^{2}(Y)\right)}|g|_{W^{2, p}\left(\mathbb{R}^{N}\right)}
$$

This, in fact, will be a consequence of Lemma 2.5, because we can represent $r_{2}^{\varepsilon}$ as follows:

$$
r_{2}^{\varepsilon}(\xi)=\int_{\mathbb{R}^{N}} \widetilde{g}_{2}^{\varepsilon}(x) e^{-i x \cdot \xi} \rho\left(\frac{x}{\varepsilon} ; \varepsilon \xi\right) d x,
$$

with

$$
\tilde{g}_{2}^{\varepsilon}(x)=\sum_{\ell \in \mathbb{Z}^{N}}\left(g(x)-g\left(x_{\ell}^{\varepsilon}\right)-\nabla g\left(x_{\ell}^{\varepsilon}\right) \cdot\left(x-x_{\ell}^{\varepsilon}\right)\right) \chi_{Y_{\ell}^{\varepsilon}}(x)
$$

which admits the following estimates:

$$
\begin{aligned}
\left\|\widetilde{g}_{2}^{\varepsilon}\right\|_{L^{2}\left(\mathbb{R}^{N}\right)} & \leq \frac{c(K)}{\left(2-\frac{N}{p}\right)} \varepsilon^{2}|g|_{W^{2, p}\left(\mathbb{R}^{N}\right)}, \\
\left\|\widetilde{g}_{2}^{\varepsilon}\right\|_{L^{p}\left(\mathbb{R}^{N}\right)} & \leq \frac{c}{\left(2-\frac{N}{p}\right)} \varepsilon^{2}|g|_{W^{2, p}\left(\mathbb{R}^{N}\right)} .
\end{aligned}
$$

As before (5.17) will be a consequence of (5.18) with $c(K)=c|K|^{1-\frac{2}{p}}$.

To establish (5.18), what we need is a generalization of Morrey's inequality for $W^{2, p}$ functions, namely

$$
\left|g(x)-g\left(x_{\ell}^{\varepsilon}\right)-\nabla g\left(x_{\ell}^{\varepsilon}\right) \cdot\left(x-x_{\ell}^{\varepsilon}\right)\right| \leq \frac{c}{\left(2-\frac{N}{p}\right)}\left|x-x_{\ell}^{\varepsilon}\right|^{2-\frac{N}{p}}|g|_{W^{2, p}\left(Y_{\ell}^{\varepsilon}\right)} \quad \forall x \in Y_{\ell}^{\varepsilon} .
$$

Admitting the above estimate, it is an easy matter to prove (5.18). But the above estimate is a consequence of Morrey's inequality for the gradient $\nabla g \in W^{1, p}\left(\mathbb{R}^{N}\right)$ and the representation

$$
g(x)-g\left(x_{\ell}^{\varepsilon}\right)-\nabla g\left(x_{\ell}^{\varepsilon}\right) \cdot\left(x-x_{\ell}^{\varepsilon}\right)=\int_{0}^{1}\left\{\nabla g\left((1-t) x_{\ell}^{\varepsilon}+t x\right)-\nabla g\left(x_{\ell}^{\varepsilon}\right)\right\} \cdot\left(x-x_{\ell}^{\varepsilon}\right) d t \quad \forall x \in Y_{\ell}^{\varepsilon} .
$$

This completes the proof of the estimate (5.14) on $r_{2}^{\varepsilon}$. Thus, as expected, $r_{2}^{\varepsilon}$ tends to zero more rapidly. The same cannot be said for the first term on the right side of (5.12). Indeed, it is equal to

$$
|Y|\left[\varepsilon^{N}\left(F^{\varepsilon} g\right)(\xi) \widetilde{\rho}^{(0)}(\varepsilon \xi)+\varepsilon^{N+1}\left(F^{\varepsilon} \frac{\partial g}{\partial x_{k}}\right)(\xi) \widetilde{\rho}^{(k)}(\varepsilon \xi)\right] .
$$

According to Lemma 2.6, we have the following convergence (apart from (5.5))

$$
\chi_{\varepsilon^{-1} Y^{\prime}}\left\{|Y|\left[\varepsilon^{N}\left(F^{\varepsilon} \frac{\partial g}{\partial x_{k}}\right)(\xi)-(2 \pi)^{N / 2} i \xi_{k} \widehat{g}(\xi)\right] \widehat{\rho}^{(k)}(\varepsilon \xi)\right\} \rightarrow 0 \quad \text { in } \quad L^{2}\left(\mathbb{R}_{\xi}^{N}\right) .
$$

This clearly allows us to complete the proof. 


\section{Proof of the main convergence results}

Applying the previously developed techniques and results, we are now in a position to prove the main convergence results stated in Section 1.2 of Introduction (namely Theorems 1.8, 1.11, and the statement (1.8)). We begin by recalling briefly the set-up. We take $f \in L^{2}\left(\mathbb{R}^{N}\right)$ and consider a sequence $u^{\varepsilon}$ satisfying (1.9), i.e.,

$$
\left\{\begin{array}{cll}
A^{\varepsilon} u^{\varepsilon}=f & \text { in } & \mathbb{R}^{N}, \\
u^{\varepsilon}-u^{*} & \text { in } & H^{1}\left(\mathbb{R}^{N}\right) \text {-weak } \\
u^{\varepsilon} \rightarrow u^{*} & \text { in } & L^{2}\left(\mathbb{R}^{N}\right) \text {-strong. }
\end{array}\right.
$$

\subsection{No concentration of energy at infinity}

Our hypothesis that $u^{\varepsilon} \rightarrow u^{*}$ in $L^{2}\left(\mathbb{R}^{N}\right)$-strong may, at first sight, look artificial. But this in not the case. If $\Omega$ is bounded and smooth, then it is classical that the weak convergence in $H^{1}(\Omega)$ will automatically imply the strong convergence in $L^{2}(\Omega)$. This is not the case in $\mathbb{R}^{N}$. To make comparisons, the correct operator to consider is $\left(A^{\varepsilon}+I\right)$ instead of $A^{\varepsilon}$ in $\mathbb{R}^{N}$. In that case, we have

Proposition 6.1 Assume that $w^{\varepsilon}$ satisfies

$$
\left\{\begin{array}{rll}
\left(A^{\varepsilon}+I\right) w^{\varepsilon}=g & \text { in } & \mathbb{R}^{N} \\
w^{\varepsilon}-w^{*} & \text { in } & H^{1}\left(\mathbb{R}^{N}\right) \text {-weak }
\end{array}\right.
$$

where $g$ is a given function in $L^{2}\left(\mathbb{R}^{N}\right)$. Then

$$
w^{\varepsilon} \rightarrow w^{*} \quad \text { in } \quad L^{2}\left(\mathbb{R}^{N}\right) \text {-strong. }
$$

Proof. First of all, following the idea of Proposition 3.1, we can neglect higher Bloch modes of $w^{\varepsilon}$ and $w^{*}$. More precisely, we can show

$$
\begin{gathered}
\int_{\varepsilon^{-1} Y_{Y^{\prime}}} \sum_{m=2}^{\infty}\left|B_{m}^{\varepsilon} w^{\varepsilon}(\xi)\right|^{2} d \xi \leq c \varepsilon^{4}, \\
\int_{\varepsilon^{-1} Y^{\prime}} \sum_{m=2}^{\infty}\left|B_{m}^{\varepsilon} w^{*}(\xi)\right|^{2} d \xi \leq c \varepsilon^{2} .
\end{gathered}
$$

Therefore, it remains to prove

$$
\int_{\varepsilon^{-1} Y^{\prime}}\left|B_{1}^{\varepsilon} w^{\varepsilon}(\xi)-B_{1}^{\varepsilon} w^{*}(\xi)\right|^{2} d \xi \rightarrow 0 .
$$

The equation in (6.2) gives the relation

$$
\left(1+\lambda_{1}^{\varepsilon}(\xi)\right) B_{1}^{\varepsilon} w^{\varepsilon}(\xi)=B_{1}^{\varepsilon} g(\xi), \quad \xi \in \varepsilon^{-1} Y^{\prime} .
$$

We use it to write

$$
\chi_{\varepsilon^{-1} Y^{\prime}}(\xi)\left(B_{1}^{\varepsilon} w^{\varepsilon}(\xi)-B_{1}^{\varepsilon} w^{*}(\xi)\right)=\chi_{\varepsilon^{-1} Y^{\prime}}(\xi) \frac{B_{1}^{\varepsilon} g(\xi)}{1+\lambda_{1}^{\varepsilon}(\xi)}-\widehat{w}^{*}(\xi)-\left(\chi_{\varepsilon^{-1} Y^{\prime}}(\xi) B_{1}^{\varepsilon} w^{*}(\xi)-\widehat{w}^{*}(\xi)\right) .
$$


According to Proposition 5.1, the last term tends to zero in $L^{2}\left(\mathbb{R}^{N}\right)$. It suffices to show

$$
\chi_{\varepsilon^{-1} Y^{\prime}}(\xi) \frac{B_{1}^{\varepsilon} g(\xi)}{1+\lambda_{1}^{\varepsilon}(\xi)}-\widehat{w}^{*}(\xi) \rightarrow 0 \quad \text { in } \quad L^{2}\left(\mathbb{R}_{\xi}^{N}\right) .
$$

Note that $w^{*}$ satisfies the homogenized equation $A^{*} w^{*}+w^{*}=g$ in $\mathbb{R}^{N}$, which is equivalent to

$$
\left(\frac{1}{2} D_{k \ell}^{2} \lambda_{1}(0) \xi_{k} \xi_{\ell}+1\right) \widehat{w}^{*}(\xi)=\widehat{g}(\xi), \quad \xi \in \mathbb{R}^{N}
$$

So, (6.4) is reduced to

$$
\chi_{\varepsilon^{-1} Y^{\prime}}(\xi) \frac{B_{1}^{\varepsilon} g(\xi)}{1+\lambda_{1}^{\varepsilon}(\xi)}-\frac{\widehat{g}(\xi)}{1+\frac{1}{2} D_{k \ell^{2}}^{2} \lambda_{1}(0) \xi_{k} \xi_{\ell}} \rightarrow 0 \quad \text { in } \quad L^{2}\left(\mathbb{R}_{\xi}^{N}\right)
$$

The above expression can be written in the form

$$
\frac{a^{\varepsilon}+b^{\varepsilon}}{c^{\varepsilon}}
$$

where

$$
\begin{aligned}
a^{\varepsilon} & =\left(1+\frac{1}{2} D_{k \ell^{2}}^{2} \lambda_{1}(0) \xi_{k} \xi_{\ell}\right)\left[\chi_{\varepsilon^{-1} Y^{\prime}}(\xi) B_{1}^{\varepsilon} g(\xi)-\widehat{g}(\xi)\right] \\
b^{\varepsilon} & =-\left(\lambda_{1}^{\varepsilon}(\xi)-\frac{1}{2} D_{k \ell}^{2} \lambda_{1}(0) \xi_{k} \xi_{\ell}\right) \widehat{g}(\xi), \\
c^{\varepsilon} & =\left(1+\lambda_{1}^{\varepsilon}(\xi)\right)\left(1+\frac{1}{2} D_{k \ell}^{2} \lambda_{1}(0) \xi_{k} \xi_{\ell}\right) .
\end{aligned}
$$

Now, we have the convergence

$$
\frac{a^{\varepsilon}}{c^{\varepsilon}}=\frac{\chi_{\varepsilon^{-1} Y^{\prime}}(\xi) B_{1}^{\varepsilon} g(\xi)-\widehat{g}(\xi)}{1+\lambda_{1}^{\varepsilon}(\xi)} \rightarrow 0 \text { in } L^{2}\left(\mathbb{R}_{\xi}^{N}\right)
$$

because $\left[1+\lambda_{1}^{\varepsilon}(\xi)\right] \geq 1$ and by the virtue of Proposition 5.1.

The convergence of $\frac{b^{\varepsilon}}{c^{\varepsilon}}$ is not immediate. To show this, we split the energy into three parts, taking $\gamma>0$ a fixed constant:

$$
\int_{\substack{|\xi| \leq \delta \varepsilon-1 \\|\xi| \leq \gamma}}\left(\frac{b^{\varepsilon}}{c^{\varepsilon}}\right)^{2} d \xi+\int_{\substack{|\xi| \leq \delta \varepsilon^{-1} \\|\xi|>\gamma}}\left(\frac{b^{\varepsilon}}{c^{\varepsilon}}\right)^{2} d \xi+\int_{\substack{|\xi|>\delta \varepsilon^{-1}\\}}\left(\frac{b^{\varepsilon}}{c^{\varepsilon}}\right)^{2} d \xi .
$$

In the first two parts, we use the estimate

$$
\left|\lambda_{1}^{\varepsilon}(\xi)-\frac{1}{2} D_{k \ell}^{2} \lambda_{1}(0) \xi_{k} \xi_{\ell}\right| \leq c|\xi|^{3} \varepsilon \quad \text { for } \quad|\xi| \leq \delta \varepsilon^{-1},
$$

which holds since $\lambda_{1}(0)=D \lambda_{1}(0)=0$ (see Proposition 1.5). In the first integral, we have $c^{\varepsilon} \geq 1$ and $\left|b^{\varepsilon}(\xi)\right| \leq c \gamma^{3} \varepsilon|\widehat{g}(\xi)|$ and consequently, it is less than

$$
c \varepsilon^{2} \int_{\mathbb{R}^{N}}|\widehat{g}(\xi)|^{2} d \xi
$$


and hence converges to zero. In the second integral, we have

$$
c^{\varepsilon} \geq \frac{1}{2} \lambda_{1}^{\varepsilon}(\xi) D_{k \ell}^{2} \lambda_{1}(0) \xi_{k} \xi_{\ell} \geq c|\xi|^{4} \geq c \gamma|\xi|^{3} \quad \text { since }|\xi| \geq \gamma>0 .
$$

As regards $b^{\varepsilon}$, we still have $\left|b^{\varepsilon}(\xi)\right| \leq c|\xi|^{3} \varepsilon|\widehat{g}(\xi)|$ and so the second integral also converges to zero.

In the third integral, we use the bounds

$$
\begin{aligned}
\left|b^{\varepsilon}\right| & \leq\left(\lambda_{1}^{\varepsilon}(\xi)+\frac{1}{2} D_{k \ell}^{2} \lambda_{1}(0) \xi_{k} \xi_{\ell}\right)|\widehat{g}(\xi)|, \\
c^{\varepsilon} & \geq \lambda_{1}^{\varepsilon}(\xi)+\frac{1}{2} D_{k \ell}^{2} \lambda_{1}(0) \xi_{k} \xi_{\ell} .
\end{aligned}
$$

Thus the third integral is estimated from above by

$$
\int_{|\xi|>\delta \varepsilon^{-1}}|\widehat{g}(\xi)|^{2} d \xi .
$$

Obviously, this tends to zero as $\varepsilon \rightarrow 0$ since $g \in L^{2}\left(\mathbb{R}^{N}\right)$.

\subsection{Corrector result in $\mathbb{R}^{N}$}

This section is devoted to the proof of Theorem 1.8 concerning the Bloch approximation $\theta^{\varepsilon}$. The proof consists of several steps which correspond to estimations of the required energy in different regions in the Fourier space (in a neighbourhood of the origin $|\eta| \leq \delta$, and in its complement $|\eta|>\delta)$.

Step 1. We decompose $u^{\varepsilon}$ as follows:

$$
u^{\varepsilon}=v^{\varepsilon}+P_{1}^{\varepsilon} u^{\varepsilon}
$$

where $v^{\varepsilon}$ and $P_{1}^{\varepsilon} u^{\varepsilon}$ are defined in (1.21) and (1.27) respectively. Thanks to Proposition 3.1, it is enough to prove

$$
\begin{aligned}
\left\|P_{1}^{\varepsilon} u^{\varepsilon}-\theta^{\varepsilon}\right\|_{L^{2}\left(\mathbb{R}^{N}\right)} & \rightarrow 0 \\
\left|P_{1}^{\varepsilon} u^{\varepsilon}-\theta^{\varepsilon}\right|_{H^{1}\left(\mathbb{R}^{N}\right)} & \leq c \varepsilon\|f\|_{L^{2}\left(\mathbb{R}^{N}\right)} .
\end{aligned}
$$

Step 2. We estimate the energies in the region $|\xi|>\delta \varepsilon^{-1}$. To this end, we introduce the quantities

$$
\begin{aligned}
\theta^{\varepsilon, \delta}(x) & =\int_{\substack{\xi \in \varepsilon^{-1} Y^{\prime} \\
|\xi|>\delta \varepsilon^{-1}}} \widehat{u}^{*}(\xi) e^{i x \cdot \xi} \phi_{1}^{\varepsilon}(x ; \xi) d \xi, \\
P_{1}^{\varepsilon, \delta} u^{\varepsilon}(x) & =\int_{\substack{\xi \in \varepsilon^{-1} Y^{\prime} \\
|\xi|>\delta \varepsilon^{-1}}} B_{1}^{\varepsilon} u^{\varepsilon}(\xi) e^{i x \cdot \xi} \phi_{1}^{\varepsilon}(x ; \xi) d \xi .
\end{aligned}
$$

We will obtain the estimates

$$
\begin{aligned}
\left\|\theta^{\varepsilon, \delta}\right\|_{L^{2}\left(\mathbb{R}^{N}\right)} & \leq c \varepsilon\|f\|_{H^{-1}\left(\mathbb{R}^{N}\right)}, \\
\left|\theta^{\varepsilon, \delta}\right|_{H^{1}\left(\mathbb{R}^{N}\right)} & \leq c \varepsilon\|f\|_{L^{2}\left(\mathbb{R}^{N}\right)}, \\
\left\|P_{1}^{\varepsilon, \delta} u^{\varepsilon}\right\|_{L^{2}\left(\mathbb{R}^{N}\right)} & \leq c \varepsilon\|f\|_{H^{-1}\left(\mathbb{R}^{N}\right)}, \\
\left|P_{1}^{\varepsilon, \delta} u^{\varepsilon}\right|_{H^{1}\left(\mathbb{R}^{N}\right)} & \leq c \varepsilon\|f\|_{L^{2}\left(\mathbb{R}^{N}\right)} .
\end{aligned}
$$


We start with (6.14). Using Lemma 2.3 with $\rho=\phi_{1}$ and the inequalities (2.5), we get

$$
\left|P_{1}^{\varepsilon, \delta} u^{\varepsilon}\right|_{H^{1}\left(\mathbb{R}^{N}\right)}^{2} \leq c \int_{\substack{\xi \in \varepsilon^{-1} Y^{\prime} \\|\xi|>\delta \varepsilon^{-1}}}\left|B_{1}^{\varepsilon} u^{\varepsilon}(\xi)\right|^{2} \lambda_{1}^{\varepsilon}(\xi) d \xi
$$

Now (6.14) easily follows if we use (1.22) and (1.24). Next, we prove (6.12). Following the above procedure, we get

$$
\left|\theta^{\varepsilon, \delta}\right|_{H^{1}\left(\mathbb{R}^{N}\right)}^{2} \leq c \int_{\substack{\xi \in \varepsilon^{-1} Y^{\prime} \\|\xi|>\delta \varepsilon^{-1}}}\left|\widehat{u}^{*}(\xi)\right|^{2}|\xi|^{2} d \xi
$$

If $f \in L^{2}\left(\mathbb{R}^{N}\right)$, then it is well-known that $u^{*} \in H^{2}\left(\mathbb{R}^{N}\right)$ and

$$
\int_{\mathbb{R}^{N}}|\xi|^{4}\left|\widehat{u}^{*}(\xi)\right|^{2} d \xi \leq c \int_{\mathbb{R}^{N}}|\widehat{f}(\xi)|^{2} d \xi
$$

Combining (6.15) and (6.16), we get easily (6.12). We now show (6.11). By Parseval identity, we have

$$
\left\|\theta^{\varepsilon, \delta}\right\|_{L^{2}\left(\mathbb{R}^{N}\right)}^{2}=\int_{\substack{\xi \in \varepsilon^{-1} Y^{\prime} \\|\xi|>\delta \varepsilon^{-1}}}\left|\widehat{u}^{*}(\xi)\right|^{2} d \xi \leq c_{\delta} \varepsilon^{2} \int_{\substack{\xi \in \varepsilon^{-1} Y^{\prime} \\|\xi|>\delta \varepsilon^{-1}}}|\xi|^{-2}|\widehat{f}(\xi)|^{2} d \xi
$$

since $u^{*}$ and $f$ are related by the homogenized equation $A^{*} u^{*}=f$ in $\mathbb{R}^{N}$. This clearly implies

$$
\left\|\theta^{\varepsilon, \delta}\right\|_{L^{2}\left(\mathbb{R}^{N}\right)}^{2} \leq c_{\delta} \varepsilon^{2} \int_{\substack{\xi \in \varepsilon^{-1} Y^{\prime} \\|\xi|>\delta \varepsilon^{-1}}}\left(1+|\xi|^{2}\right)^{-1}|\widehat{f}(\xi)|^{2} d \xi=c_{\delta} \varepsilon^{2}\|f\|_{H^{-1}\left(\mathbb{R}^{N}\right)}^{2} .
$$

The proof of (6.13) is completely analogous.

Step 3. Now, we consider the energies in $|\xi| \leq \delta \varepsilon^{-1}$. To this end, let us define

$$
\omega^{\varepsilon}(x)=\int_{|\xi| \leq \delta \varepsilon^{-1}}\left(B_{1}^{\varepsilon} u^{\varepsilon}(\xi)-\widehat{u}^{*}(\xi)\right) e^{i x \cdot \xi} \phi_{1}^{\varepsilon}(x ; \xi) d \xi
$$

and show that

$$
\begin{aligned}
\left\|\omega^{\varepsilon}\right\|_{L^{2}\left(\mathbb{R}^{N}\right)} & \rightarrow 0, \\
\left|\omega^{\varepsilon}\right|_{H^{1}\left(\mathbb{R}^{N}\right)} & \leq c \varepsilon\|f\|_{L^{2}\left(\mathbb{R}^{N}\right)} .
\end{aligned}
$$

To prove (6.18), we decompose the integrand as follows:

$$
B_{1}^{\varepsilon} u^{\varepsilon}-\widehat{u}^{*}=B_{1}^{\varepsilon}\left(u^{\varepsilon}-\widehat{u}^{*}\right)+\left(B_{1}^{\varepsilon} u^{*}-\widehat{u}^{*}\right) .
$$

By Parseval equality, the first term in $L^{2}$-norm is bounded above by $\left\|u^{\varepsilon}-u^{*}\right\|_{L^{2}\left(\mathbb{R}^{N}\right)}$ which, by our hypothesis, converges to zero. That the second term converges to zero in $L^{2}\left(\mathbb{R}^{N}\right)$ is proved in Proposition 5.1. 
Next, we turn are attention to the proof of (6.19). By Lemma 2.1, we have

$$
\left|\omega^{\varepsilon}\right|_{H^{1}\left(\mathbb{R}^{N}\right)}^{2} \leq c \int_{|\xi| \leq \delta \varepsilon^{-1}} \lambda_{1}^{\varepsilon}(\xi)\left|B_{1}^{\varepsilon} u^{\varepsilon}(\xi)-\widehat{u}^{*}(\xi)\right|^{2} d \xi .
$$

To estimate the above integral, we write the integrand as

$$
B_{1}^{\varepsilon} u^{\varepsilon}(\xi)-\widehat{u}^{*}(\xi)=\lambda_{1}^{\varepsilon}(\xi)^{-1}\left(B_{1}^{\varepsilon} f(\xi)-\widehat{f}(\xi)\right)+\left[\lambda_{1}^{\varepsilon}(\xi)^{-1}-\left(\frac{1}{2} D_{k \ell}^{2} \lambda_{1}(0) \xi_{k} \xi_{\ell}\right)^{-1}\right] \widehat{f}(\xi)
$$

Thus we get, using (1.24), that

$$
\left\{\begin{aligned}
\left|\omega^{\varepsilon}\right|_{H^{1}\left(\mathbb{R}^{N}\right)}^{2} \leq & c \int_{|\xi| \leq \delta \varepsilon} \frac{\left|B_{1}^{\varepsilon} f(\xi)-\widehat{f}(\xi)\right|^{2}}{|\xi|^{2}} d \xi+ \\
& +c \int_{|\xi| \leq \delta \varepsilon^{-1}} \lambda_{1}^{\varepsilon}(\xi)\left|\lambda_{1}^{\varepsilon}(\xi)^{-1}-\left(\frac{1}{2} D_{k \ell^{2}}^{2} \lambda_{1}(0) \xi_{k} \xi_{\ell}\right)^{-1}\right|^{2}|\widehat{f}(\xi)|^{2} d \xi
\end{aligned}\right.
$$

To estimate the first term on the right hand side of (6.21), we represent the integrand as

$$
\frac{B_{1}^{\varepsilon} f(\xi)-\widehat{f}(\xi)}{|\xi|}=\int_{\mathbb{R}^{N}} f(x) e^{-i x \cdot \xi} \frac{\left(\phi_{1}^{\varepsilon}(x ; \xi)-\phi_{1}^{\varepsilon}(x ; 0)\right)}{|\xi|} d x .
$$

Applying Lemma 2.5, and using $\left\|\phi_{1}(\cdot ; \eta)-\phi_{1}(\cdot ; 0)\right\|_{L^{2}(Y)} \leq c|\eta|$ for $|\eta| \leq \delta$, we get

$$
\int_{|\xi| \leq \delta \varepsilon^{-1}} \frac{\left|B_{1}^{\varepsilon} f(\xi)-\widehat{f}(\xi)\right|^{2}}{|\xi|^{2}} d \xi \leq c \varepsilon^{2}\|f\|_{L^{2}\left(\mathbb{R}^{N}\right)}^{2}
$$

The second term on the right side of (6.21) can be rewritten, using homogenized equation as

$$
\int_{|\xi| \leq \delta \varepsilon^{-1}} \frac{\left|\lambda_{1}^{\varepsilon}(\xi)-\frac{1}{2} D_{k \ell}^{2} \lambda_{1}(0) \xi_{k} \xi_{\ell}\right|^{2}}{\lambda_{1}^{\varepsilon}(\xi)}\left|\widehat{u}^{*}(\xi)\right|^{2} d \xi .
$$

Using (6.6) and (1.24), we see that the above integral is estimated from above by

$$
c \varepsilon^{2} \int_{|\xi| \leq \delta \varepsilon^{-1}}|\xi|^{4}\left|\widehat{u}^{*}(\xi)\right|^{2} d \xi \leq c \varepsilon^{2}\|f\|_{L^{2}\left(\mathbb{R}^{N}\right)}^{2} .
$$

This establishes (6.19) and hence the result.

\subsection{Asymptotic expansion of the Bloch approximation}

In this concluding paragraph, we prove Theorem 1.11.

Proof of (i) We have the following decomposition:

$$
\theta^{\varepsilon}(x)-u^{*}(x)=z^{\varepsilon}(x)+\theta^{\varepsilon, \delta}(x)+u^{*, \delta}(x),
$$


where

$$
\begin{aligned}
z^{\varepsilon}(x) & =\int_{\substack{\xi \in \varepsilon^{-1} Y^{\prime} \\
|\xi| \leq \delta \varepsilon^{-1}}} \widehat{u}^{*}(\xi) e^{i x \cdot \xi}\left(\phi_{1}^{\varepsilon}(x ; \xi)-\phi_{1}^{\varepsilon}(x ; 0)\right) d \xi, \\
u^{*, \delta}(x) & =\frac{1}{(2 \pi)^{N / 2}} \int_{|\xi|>\delta \varepsilon^{-1}} \widehat{u}^{*}(\xi) e^{i x \cdot \xi} d \xi,
\end{aligned}
$$

and $\theta^{\varepsilon, \delta}$ is defined in (6.9).

The second term has already been estimated in $L^{2}$-norm (see (6.11)). The same proof shows that the third term admits a bound

$$
\left\|u^{*, \delta}\right\|_{L^{2}\left(\mathbb{R}^{N}\right)} \leq c \varepsilon\|f\|_{H^{-1}\left(\mathbb{R}^{N}\right)} \leq c \varepsilon\left\|u^{*}\right\|_{H^{1}\left(\mathbb{R}^{N}\right)} .
$$

To estimate the first term on the right side of (6.22), we must proceed differently. In fact, it is essential to use Lemma 2.3. We see then that

$$
\left\|z^{\varepsilon}\right\|_{L^{2}\left(\mathbb{R}^{N}\right)}^{2}=\int_{\substack{\xi \in \varepsilon-1 Y^{\prime} \\|\xi| \leq \delta \varepsilon^{-1}}}\left|\widehat{u}^{*}(\xi)\right|^{2}\left\|\phi_{1}^{\varepsilon}(\cdot ; \xi)-\phi_{1}^{\varepsilon}(\cdot ; 0)\right\|_{L^{2}(Y)}^{2} d \xi .
$$

Using the Lipschitz continuity of the map $\eta \mapsto \phi_{1}(\cdot ; \eta) \in L^{2}(Y)$, for $|\eta| \leq \delta$, we see that the above integral can be majorized, and we obtain

$$
\left\|z^{\varepsilon}\right\|_{L^{2}\left(\mathbb{R}^{N}\right)}^{2} \leq c \varepsilon^{2} \int_{|\xi| \leq \delta \varepsilon^{-1}}\left|\widehat{u}^{*}(\xi)\right|^{2}|\xi|^{2} d \xi \leq c \varepsilon^{2}\left|u^{*}\right|_{H^{1}\left(\mathbb{R}^{N}\right)}^{2} .
$$

This finishes the proof of (i). We note that we cannot, in general, assert that

$$
\left|u^{*}\right|_{H^{1}\left(\mathbb{R}^{N}\right)} \leq c\|f\|_{H^{-1}\left(\mathbb{R}^{N}\right)}
$$

as we are working on the entire space $\mathbb{R}^{N}$.

Proof of (ii) Because of (i), it suffices to prove

$$
\left|\theta^{\varepsilon}-u^{*}-\varepsilon \chi_{k}^{\varepsilon} \frac{\partial u^{*}}{\partial x_{k}}\right|_{H^{1}\left(\mathbb{R}^{N}\right)} \leq c \varepsilon\|f\|_{L^{2}\left(\mathbb{R}^{N}\right)} .
$$

To this end, we use once again the decomposition (6.22) for $\left(\theta^{\varepsilon}-u^{*}\right)$ in terms of $z^{\varepsilon}, \theta^{\varepsilon, \delta}$, and $u^{*, \delta}$. For $\theta^{\varepsilon, \delta}$, we have the estimate $(6.12)$. For $u^{*, \delta}$, we can easily derive the estimate

$$
\left|u^{*, \delta}\right|_{H^{1}\left(\mathbb{R}^{N}\right)}^{2} \leq c \int_{|\xi|>\delta \varepsilon^{-1}}|\xi|^{2}\left|\widehat{u}^{*}(\xi)\right|^{2} d \xi \leq c_{\delta} \varepsilon^{2}\|f\|_{L^{2}\left(\mathbb{R}^{N}\right)}^{2} .
$$

Thus, we are reduced to obtain the estimate

$$
\left|z^{\varepsilon}-\varepsilon \chi_{k}^{\varepsilon} \frac{\partial u^{*}}{\partial x_{k}}\right|_{H^{1}\left(\mathbb{R}^{N}\right)} \leq c \varepsilon\|f\|_{L^{2}\left(\mathbb{R}^{N}\right)} .
$$

To this end, we use the representation

$$
\frac{\partial u^{*}}{\partial x_{k}}(x)=\frac{1}{(2 \pi)^{N / 2}} \int_{\mathbb{R}^{N}}\left(i \xi_{k}\right) \widehat{u}^{*}(\xi) e^{i x \cdot \xi} d \xi,
$$


and combine it with the representation $(6.23)$ for $z^{\varepsilon}$. We get

$$
\begin{aligned}
z^{\varepsilon}(x)-\varepsilon \chi_{k}^{\varepsilon}(x) \frac{\partial u^{*}}{\partial x_{k}}(x) & =\int_{|\xi| \leq \delta \varepsilon^{-1}} \widehat{u}^{*}(\xi) e^{i x \cdot \xi}\left(\phi_{1}^{\varepsilon}(x ; \xi)-\phi_{1}^{\varepsilon}(x ; 0)-i p^{(0)} \chi_{k}^{\varepsilon}(x) \varepsilon \xi_{k}\right) d \xi- \\
& -\int_{|\xi|>\delta \varepsilon^{-1}} i p^{(0)} \chi_{k}^{\varepsilon}(x) \varepsilon \xi_{k} \widehat{u}^{*}(\xi) e^{i x \cdot \xi} d \xi .
\end{aligned}
$$

To estimate the first term on the right side of (6.30), we appeal to Lemma 2.3. Further, we use

$$
\left\|\phi_{1}(\cdot ; \eta)-\phi_{1}(\cdot ; 0)-i p^{(0)} \chi_{k}(\cdot) \eta_{k}\right\|_{H^{1}(Y)} \leq c|\eta|^{2} \quad \text { for } \quad|\eta| \leq \delta .
$$

The estimate on the second term on the right side of (6.30) is more straightforward. We get finally

$$
\left|z^{\varepsilon}-\varepsilon \chi_{k}^{\varepsilon} \frac{\partial u^{*}}{\partial x_{k}}\right|_{H^{1}\left(\mathbb{R}^{N}\right)}^{2} \leq c \varepsilon^{2} \int_{\mathbb{R}^{N}}|\xi|^{4}\left|\widehat{u}^{*}(\xi)\right|^{2} d \xi .
$$

This completes the proof of (6.29) and hence (ii).

Proof of (iii) Consider again the decomposition (6.22). Thanks to (6.9) and (6.15), we have the estimates

$$
\begin{aligned}
\left\|\theta^{\varepsilon, \delta}\right\|_{L^{2}\left(\mathbb{R}^{N}\right)} & \leq c \varepsilon^{2}\|f\|_{L^{2}\left(\mathbb{R}^{N}\right)}, \\
\left|\theta^{\varepsilon, \delta}\right|_{H^{1}\left(\mathbb{R}^{N}\right)} & \leq c \varepsilon^{2}|f|_{H^{1}\left(\mathbb{R}^{N}\right)} .
\end{aligned}
$$

Similar techniques imply

$$
\begin{aligned}
\left\|u^{*, \delta}\right\|_{L^{2}\left(\mathbb{R}^{N}\right)} & \leq c \varepsilon^{2}\|f\|_{L^{2}\left(\mathbb{R}^{N}\right)}, \\
\left|u^{*, \delta}\right|_{H^{1}\left(\mathbb{R}^{N}\right)} & \leq c \varepsilon^{2}|f|_{H^{1}\left(\mathbb{R}^{N}\right)} .
\end{aligned}
$$

On the other hand, it is clear from the representation (6.30) that

$$
\left\|z^{\varepsilon}-\varepsilon \chi_{k}^{\varepsilon} \frac{\partial u^{*}}{\partial x_{k}}\right\|_{L^{2}\left(\mathbb{R}^{N}\right)} \leq c \varepsilon^{2}\|f\|_{L^{2}\left(\mathbb{R}^{N}\right)} .
$$

Thus, it is enough to obtain the estimate

$$
\left|\theta^{\varepsilon}-u^{*}-\varepsilon \chi_{k}^{\varepsilon} \frac{\partial u^{*}}{\partial x_{k}}+\varepsilon^{2}\left(\chi_{k \ell}^{\varepsilon}+\beta_{k \ell}^{(2)}\right) \frac{\partial^{2} u^{*}}{\partial x_{k} \partial x_{\ell}}\right|_{H^{1}\left(\mathbb{R}^{N}\right)} \leq c \varepsilon^{2}|f|_{H^{1}\left(\mathbb{R}^{N}\right)} .
$$

Thanks to (6.33) and (6.35), we are reduced to showing that

$$
\left|z^{\varepsilon}-\varepsilon \chi_{k}^{\varepsilon} \frac{\partial u^{*}}{\partial x_{k}}+\varepsilon^{2}\left(\chi_{k \ell}^{\varepsilon}+\beta_{k \ell}^{(2)}\right) \frac{\partial^{2} u^{*}}{\partial x_{k} \partial x_{\ell}}\right|_{H^{1}\left(\mathbb{R}^{N}\right)} \leq c \varepsilon^{2}|f|_{H^{1}\left(\mathbb{R}^{N}\right)} .
$$

We can write

$$
\begin{aligned}
z^{\varepsilon}(x) & -\varepsilon \chi_{k}^{\varepsilon}(x) \frac{\partial u^{*}}{\partial x_{k}}(x)+\varepsilon^{2}\left(\chi_{k \ell}^{\varepsilon}(x)+\beta_{k \ell}^{(2)}\right) \frac{\partial^{2} u^{*}}{\partial x_{k} \partial x_{\ell}}(x)= \\
& =\int_{|\xi| \leq \delta \varepsilon^{-1}} \widehat{u}^{*}(\xi) e^{i x \cdot \xi}\left[\phi_{1}^{\varepsilon}(x ; \xi)-\phi_{1}^{\varepsilon}(x ; 0)-i p^{(0)} \chi_{k}^{\varepsilon}(x) \varepsilon \xi_{k}+p^{(0)}\left(\chi_{k \ell}^{\varepsilon}(x)+\beta_{k \ell}^{(2)}\right) \varepsilon^{2} \xi_{k} \xi_{\ell}\right] d \xi- \\
(6.39) & -\int_{|\xi|>\delta \varepsilon^{-1}} i p^{(0)} \chi_{k}^{\varepsilon}(x) \varepsilon \xi_{k} \widehat{u}^{*}(\xi) e^{i x \cdot \xi} d \xi+\int_{|\xi|>\delta \varepsilon^{-1}} p^{(0)}\left(\chi_{k \ell}^{\varepsilon}(x)+\beta_{k \ell}^{(2)}\right) \varepsilon^{2} \xi_{k} \xi_{\ell} \widehat{u}^{*}(\xi) e^{i x \cdot \xi} d \xi .
\end{aligned}
$$


The analysis of the right hand side of (6.39) is similar to that of (6.30). The new information needed is the following:

$(6.40)\left\|\phi_{1}(\cdot ; \eta)-\phi_{1}(\cdot ; 0)-i p^{(0)} \chi_{k}(\cdot) \eta_{k}+p^{(0)}\left(\chi_{k \ell}(\cdot)+\beta_{k \ell}^{(2)}\right) \eta_{k} \eta_{\ell}\right\|_{H^{1}(Y)} \leq c|\eta|^{3}$ for $|\eta| \leq \delta$,

which is a simple consequence of Proposition 1.10. The proof is concluded via a simple application of Lemma 2.3 .

\section{Acknowledgements}

This research was done thanks to a couple of visits of R. Orive and M. Vanninathan to the Center of Mathematical Modelling and to the Department of Mathematical Engineering of the University of Chile, supported by the Fondap's Programme on Mathematical-Mechanics. These authors thank warmly these Institutions.

The second author was supported by a doctoral fellowship of the "Comunidad Autonoma de Madrid" (SPAIN), and partially by grant PB96-0663 of the DGES (SPAIN) and the TMR project of the EU TMR Network "Homogenization and Multiple Scales".

\section{References}

[1] A. Bensoussan, J. L. Lions, and G. Papanicolaou, Asymptotic Analysis for Periodic Structures, North-Holland, Amsterdam, 1978.

[2] H. BrezIs, Analyse Fonctionnelle, Théorie et Applications, Collection Mathématiques Appliquées pour la Maîtrise, Masson, Paris, 1983.

[3] C. Conca, R. Orive, and M. Vanninathan, Bloch approximation in bounded domains, working paper, (2001).

[4] C. Conca, J. Planchard, and M. Vanninathan, Fluids and Periodic Structures, no. 38 in Research in Applied Mathematics, J. Wiley/Masson, New York/Paris, 1995.

[5] C. Conca and M. Vanninathan, Homogenization of periodic structures via Bloch decomposition, SIAM J. Appl. Math., 57 (1997), pp. 1639-1659.

[6] - On uniform $\mathrm{H}^{2}$-estimates in periodic homogenization, Proc. Roy. Soc. Edinburgh Sect. A, (2000).

[7] F. Santosa And W. W. Symes, A dispersive effective medium for wave propagation in periodic composites, SIAM J. Appl. Math., 51 (1991), pp. 984-1005.

[8] L. TARTAR, Problèmes d'homogénéisation dans les équations aux dérivées partielles, in Cours Peccot au Collège de France, 1977. 